\title{
Update of early phase clinical trials in cancer immunotherapy
}

\author{
Dae Ho Lee ${ }^{*}$ \\ Department of Oncology, University of Ulsan College of Medicine, Asan Medical Center, Seoul 05505, Korea
}

\begin{abstract}
Immunotherapy has revolutionized the landscape of cancer treatment and become a standard pillar of the treatment. The two main drivers, immune checkpoint inhibitors and chimeric antigen receptor T cells, contributed to this unprecedented success. However, despite the striking clinical improvements, most patients still suffer from disease progression because of the evolution of primary or acquired resistance. This mini-review summarizes new treatment options including novel targets and interesting combinational approaches to increase our understanding of the mechanisms of the action of and resistance to immunotherapy, to expand our knowledge of advances in biomarker and therapeutics development, and to help to find the most appropriate option or a way of overcoming the resistance for cancer patients. [BMB Reports 2021; 54(1): 70-88]
\end{abstract}

\section{INTRODUCTION}

During the last decade, immunotherapy has become a standard pillar of cancer treatment with already existing pillars of surgery, radiation, cytotoxic chemotherapy, and molecular-targeted therapy. Two main derivers have contributed to this unprecedented success; one is immune checkpoint (IC) inhibitors and the other is chimeric antigen receptor (CAR) T cells. ICs, such as cytotoxic T-lymphocyte-associated protein (CTLA-4) and programed cell-death protein-1/programmed cell-death protein ligand-1 (PD-1/PD-L1), are exploited by cancer cells to evade host immunity, and their blocking monoclonal antibodies can restore or reinvigorate the host immunity. At first, the disruption of the pathway was shown to induce durable remission or even cures in patients with advanced or metastatic melanoma or Non-small cell lung cancer (NSCLC). More success has followed in different tumor types, including renal cell carcinoma (RCC) and urothelial tumors, and in different clinical situations, including adjuvant therapy after surgery, consolidation therapy after chemoradio-

*Corresponding author. Tel: +82-2-3010-3214; Fax: +82-2-3010-6961; E-mail: leedaeho@amc.seoul.kr

https://doi.org/10.5483/BMBRep.2021.54.1.242

Received 19 October 2020, Revised 26 November 2020, Accepted 15 December 2020

Keywords: Cancer immunotherapy, Clinical trials, Novel biomarkers, Novel therapies, Preclinical study therapy, and even in neo-adjuvant therapy before surgery. On the other hand, CAR-T cells also showed very impressive clinical outcomes in hematologic malignancies despite their specific life-threatening toxicities. Two CAR-T cell therapeutics, tisagenlecleucel and axicabtagen-ciloleucel, were approved by the US FDA and EMA for acute lymphoblastic leukemia (ALL) and diffuse large B-cell lymphoma (DLBCL). In fact, CAR-T cells are different from IC inhibitors in that they are genetically engineered $T$ cells, whereas IC inhibitors are a kind of classical monoclonal antibodies, giving different technical, regulatory, and economic challenges.

Immunotherapy can be categorized into 'passive' or 'active'. The former is to give directly immune molecules that can kill tumor cells, such as specific tumor molecule-targeting monoclonal antibodies or immune cells, such as CAR-T cells or CAR-NK cells. The latter is to give patients molecules that can activate their own immune system, including cytokines such as IFN-gamma or IL-2, cancer vaccines and immunomodulators, such as IC inhibitors or other co-stimulatory agonists, which finally kill tumor cells indirectly. The movement of CAR-T cells toward solid tumors was sometimes blocked by the lack of appropriately identified cancer-specific antigens, meaning that passive immunotherapy needs cancer-specific antigens or suitable targets. On the other hand, the success of IC therapy did not always repeat in all patients, because of difference in individuals' immune responses. As a result, many patients do not respond to IC inhibitors at all, or some patients may lose their initial responsiveness during their treatment, perhaps because of a failure to provoke or maintain the host immunity, or perhaps partly because of a defect of their own immune system itself.

This review focuses mainly on clinical and some pre-clinical studies of immunotherapy, especially targeting immune molecules, other than passive or adoptive immunotherapy and cancer vaccines, considering that they have rather different or unique challenges. However, an improved understanding of immunotherapy might help to create new therapeutic approaches or optimize the therapeutic options including CAR-T cells or cancer vaccines.

\section{CO-INHIBITORY IMMUNE CHECKPOINT INHIBITORS OR ANTAGONISTS (Table 1)}

Currently approved IC inhibitors target CTLA-4 and PD-1/PD-L1 co-inhibitory pathways. However, as mentioned above, despite their continuing success, most patients are still unresponsive 
Table 1. Co-inhibitory immune checkpoint inhibitors or antagonists

\begin{tabular}{|c|c|c|c|c|}
\hline Target & Agent & Company & $\begin{array}{l}\text { Clinical } \\
\text { phase }\end{array}$ & Findings \\
\hline \multirow[t]{10}{*}{ TIGIT } & $\begin{array}{l}\text { Tiragolumab } \\
\text { (MTIG7192A) }\end{array}$ & Roche & $\mathrm{II} / \mathrm{III}$ & $\begin{array}{l}\text { - Phase I trial } \\
\text { - Monotherapy: ORR 0\% } \\
\text { - Tiragolumab/atezolizumab for NSCLC: ORR 46\% \& DCR 85\% } \\
\text { - Phase II trial of tiragolumab/ atezolizumab } \\
\text { - All NSCLC, ORR } 37 \% \text { \& mPFS } 5.6 \text { months } \\
\text { (HR 0.58, 95\% Cl 0.38-0.89) } \\
\text { - High PD-L1 ( } \geq 50 \% \text { ), ORR 66\% \& mPFS not reached } \\
\text { (HR 0.30, 95\% Cl 0.15-0.61) }\end{array}$ \\
\hline & Vibostolimab (MK-7684) & Merck Sharp \& Dohme & II & $\begin{array}{l}\text { - Phase I trial } \\
\text { - Monotherapy: ORR 7\% } \\
\text { - Vibostolimab/pembrolizumab: ORR 5\% } \\
\text { - Phase I part B for anti-PD-1/PD-L1 therapy-naïve patients: } \\
\text { - ORR } 29 \% \text { \& mPFS } 5.4 \text { mo } \\
\text { - PD-L1 } 21 \% \text {, ORR } 46 \% \text { \& mPFS } 8.4 \text { mo } \\
\text { - PD-L1 }<1 \% \text {, ORR } 25 \% \text { \& mFS } 4.1 \text { mo }\end{array}$ \\
\hline & BMS-986207 & Bristol-Myers Squibb & $\mathrm{I} / \mathrm{II}$ & $\bullet \pm$ Nivolumab \\
\hline & ASP8374 & Astellas & I & - \pm Pembrolizumab \\
\hline & AB154 & Arcus Bioscience & $\mathrm{I} / \mathrm{II}$ & $\begin{array}{l}\text { - Zimberelimab (AB122, anti-PD-1) vs } \\
\text { zimberelimab+ANB154 vs } \\
\text { zimberelimab+ANB154+AB928 } \\
\text { (dual adenosine receptor antagonist) }\end{array}$ \\
\hline & BGB-A1217 & Beigene & I & $\bullet+$ Tislelizumab (anti-PD-1) \\
\hline & Eigliimab (OMP-313M32) & $\begin{array}{l}\text { Mereo BioPharma } \\
\text { (OncoMed } \\
\text { Pharmaceuticals) }\end{array}$ & I & $\bullet \pm$ Nivolumab \\
\hline & COM902 & Compugen & I & \\
\hline & IBI939 & Innovent Biologics & I & \\
\hline & EOS884448 & iTeos Therapeutics & I & \\
\hline \multirow[t]{11}{*}{ LAG-3 } & Relatlimab (BMS-986016) & Bristol-Myers Squibb & II & $\begin{array}{l}\text { - Relatlimab/nivolumab for melanoma, } \\
\text { ORR } 11.5 \% \text { \& DCR } 49 \% \\
\text { - LAG-3 } \geq 1 \% \text {, ORR } 18 \% \text { \& DCR } 64 \%\end{array}$ \\
\hline & $\begin{array}{l}\text { Eftilagimod alpha } \\
\text { (IMP321) }\end{array}$ & Immutep & II & $\begin{array}{l}\text { - Eftilagimod/pembrolizumab } \\
\text { for NSCLC as first-line, ORR 53\% } \\
\text { for HNSCC as second-line, ORR 39\% }\end{array}$ \\
\hline & $\begin{array}{l}\text { Leramilimab } \\
\text { (LAG525/IMP701) }\end{array}$ & Novartis & II & $\begin{array}{l}\text { - Leramilimab/spartalizumab } \\
\text { - For mesothelioma, 25\% (2/8) } \\
\text { - For TNBC, } 40 \%(2 / 5) \\
\text { - } \pm \text { NIR178 } \pm \text { canakinumab }\end{array}$ \\
\hline & MK-4280 & Merck Sharp \& Dohme & II & $\begin{array}{l}\text { Phase I trial } \\
\text { - monotherapy: ORR 6\% \& DCR 17\% } \\
\text { - MK-4280/pembrolizumab: ORR 27\% \& DCR 40\% }\end{array}$ \\
\hline & Fianlimab (REGN3767) & Regeneron & III & • + Cemiplimab (REGN2810, Anti-PD-1) \\
\hline & TSR-033 & Tesaro & I & $\bullet \pm$ Dostarlimab (TSR-042, anti-PD-1) \\
\hline & BI-754111 & Boehringer Ingelheim & I & $\begin{array}{l}\bullet \pm \mathrm{BI}-754091 \text { (anti-PD-1) } \\
\cdot \mathrm{BI}-754091 \pm \mathrm{BI}-754111\end{array}$ \\
\hline & Sym-022 & Symphogen & I & - Sym-021 (anti-PD-1) \pm Sym-022 \\
\hline & INCAGN02385 & Incyte & $\mathrm{I} / \mathrm{II}$ & $\begin{array}{l}\bullet+\text { + INCAGN02390 (anti-TIM3) } \\
\bullet \text { + INCAGN02390/INCMGA00012 (anti-PD-1) }\end{array}$ \\
\hline & Tebotelimab (MGD013) & MacorGenics & I & - \pm Brivanib (VEGFR2 inhibitor) or margetuximab (anti-HER2) \\
\hline & RO7247669 & Roche & I & \\
\hline \multirow[t]{3}{*}{ TIM-3 } & Cobolimab (TSR-022) & Tesaro & I & $\begin{array}{l}\text { - Combination with dostarlimab; } \\
\text { - ORR } 15 \% \text { \& DCR } 40 \% \\
\text { - TSR-022 } \pm \text { TSR-042 or nivolumab } \pm \text { TSR-033 or docetaxel }\end{array}$ \\
\hline & MBG453 & Novartis & $\mathrm{I} / \mathrm{II}$ & $\bullet \pm$ Spartalizumab \\
\hline & Sym023 & Symphogen & I & - Sym-021 (anti-PD-1) \pm Sym-023 \\
\hline
\end{tabular}


Table 1. Continued

\begin{tabular}{|c|c|c|c|c|}
\hline Target & Agent & Company & $\begin{array}{l}\text { Clinical } \\
\text { phase }\end{array}$ & Findings \\
\hline & SHR1702 & $\begin{array}{l}\text { Jiangsu HengRui } \\
\text { Medicine }\end{array}$ & I & $\bullet \pm$ Camrelizumab (SHR-1210, anti-PD-1) \\
\hline & LY3321367 & Eli Lilly & I & $\bullet \pm$ LY3300054 (Anti-PD-L1) \\
\hline & BMS986258 & Bristol-Myers Squibb & $1 / I I$ & $\bullet \pm$ Nivolumab \\
\hline & INCAGN2390 & Incyte & I & $\begin{array}{l}\bullet+\text { + INCAGN02385 (anti-LAG3) } \\
\text { - + INCAGN02385/INCMGA00012 }\end{array}$ \\
\hline & RO7121661 & Roche & I & \\
\hline \multirow[t]{3}{*}{$\begin{array}{l}\text { VISTA } \\
\text { (B7-H5) }\end{array}$} & CA-170 & Curis & II & $\begin{array}{l}\text { - For Hodgkin disease: CBR 52\% } \\
\text { - For NSCLC; ORR 0\% }\end{array}$ \\
\hline & JNJ-61610588 & Janssen & I & \\
\hline & HMBD-002 & $\begin{array}{l}\text { Hummingbird } \\
\text { Bioscience }\end{array}$ & I & \\
\hline \multirow[t]{3}{*}{$\begin{array}{l}\mathrm{B} 7-\mathrm{H} 3 \\
(\mathrm{CD} 276)\end{array}$} & $\begin{array}{l}\text { Enoblituzumab } \\
\text { (MGA271) }\end{array}$ & MacroGenics & II & $\begin{array}{l}\text { • Enoblituzumab/pembrolizumab } \\
\text { - Anti-PD1/PD-L1 naïve patients } \\
\text { NSCLC: ORR 35.7\% \& DCR 92.9\% } \\
\text { HNSCC: ORR 33.3\% \& DCR 61.1\% } \\
\text { - Naïve patients with B7-H3 } \geq 10 \% \\
\text { NSCLC: ORR 45.5\% \& DCR 90.9\% } \\
\text { HNSCC: ORR 40.0\% \& DCR } 73.3 \%\end{array}$ \\
\hline & MGD009 & MacroGenics & I & - Partial clinical hold due to hepatotoxicity \\
\hline & Omburtamab $(8 \mathrm{H} 9)$ & Y-mAbs Therapeutic & $\mathrm{II} / \mathrm{III}$ & \\
\hline BTLA & TAB004/JS004 & $\begin{array}{l}\text { Shanghai Junshi } \\
\text { Bioscience }\end{array}$ & I & \\
\hline
\end{tabular}

BTLA: B- and T-lymphocyte attenuator, DCR: disease-control rate, HNSCC: head and neck squamous cell carcinoma, LAG-3: Lymphocyte activation gene-3, NSCLC: Non-small cell lung cancer, mo: month, mPFS: median progression-free survival, ORR: overall response rate, PD-1: programed cell death protein-1, PD-L1: programmed cell death protein ligand-1, TIGIT: T cell immunoglobulin and ITIM domain, TIM-3: transmembrane immunoglobulin and mucin domain 3, TNBC: triple negative breast cancer, VISTA: V-domain Ig Suppressor of T-cell Activation.

and show intolerable immune-mediated toxicity or disease progression in their treatment. Therefore, interest has grown towards other co-inhibitory ICs to overcome their refractoriness or resistance. The front runners include co-inhibitory ICs co-expressing PD-1/CTLA-4, such as LAG-3, TIM-3, and TIGIT, which are highly expressed on dysfunctional or exhausted T cells (1, 2). Many agents targeting one of them have been explored as monotherapy, but their efficacies were not as good as those of anti-PD-1/PD-L1 monotherapy. They are often investigated in combination with anti-PD-1/PD-L1, especially based on preclinical studies which showed that combinational receptor blockade has strong synergistic effects and results in improved effector $\mathrm{CD}^{+}{ }^{\mathrm{T}}$ cell and Natural Killer (NK) cell function as well as decreased regulatory $\mathrm{T}$ cell (Treg)-mediated suppression. Other co-inhibitory ICs, such as VISTA, B7-H3 and BTLA, are also being explored. However, because their roles in tumors showing primary or acquired resistance are not known well yet and need to be elucidated further, a few agents are being investigated under early clinical development.

\section{T cell immunoglobulin and ITIM domain (TIGIT)}

TIGIT is an Ig-like protein homologous to CD28 interfering with the co-stimulatory axis of the CTLA-4/B7/CD28 axis and plays a key role in the suppression of T-cell proliferation and activation and promotion of T-cell exhaustion. In fact, TIGIT inhibits innate and adaptive immunity by means of, multiple mechanisms, including direct inhibition of $T$ cells through binding CD155 on T cells, indirect inhibition of T cells via increasing interleukin (IL)-10 production, decreasing IL-12 production by binding CD155 on antigen-presenting cells (APCs), and increasing immunosuppressive functions of Tregs. NK cells are also inhibited by TIGIT (3). In mice, dual TIGIT and PD-1/ PD-L1 blockade synergize to increase the proliferation and function of antitumor $\mathrm{CD}^{+}{ }^{+} \mathrm{T}$ cells, resulting in prolonged overall survival (4). In melanoma patients, dual PD-1/TIGIT blockade also increases the proliferation and function of tumor antigenspecific $\mathrm{CD}^{+} \mathrm{T}$ cells and tumor-infiltrating lymphocytes (TILs) when compared to single blockade (5).

Tiragolumab (MTIG7192A) showed good tolerability as both monotherapy and combinational therapy with atezolizumab, an anti-PD-L1 antibody, and preliminary but promising clinical activity in a phase I trial. In the trial, no response was observed from monotherapy, although the patients included were known to be resistant; however, out of 13 NSCLC patients receiving the combination, the response rate (RR) was $46 \%$ and diseasecontrol rate (DCR) was $85 \%$ (6). In a following randomized 
phase II CITYSCAPE trial, an atezolizumab/tiragolumab combination compared with atezolizumab alone resulted in improved progression-free survival (PFS) with medians of 5.6 months versus 3.9 months, respectively, or $42 \%$ reduction in the risk of disease progression or death (stratified hazard ratio [HR], 0.58; 95\% confidence interval [Cl], 0.38-0.89). Overall response rate (ORR) was also almost doubled, from $21 \%$ to $37 \%$. Of more note, explorative analysis did show $70 \%$ reduction in the risk in patients with PD-L1 expression $\geq 50 \%$ (stratified HR, $0.30 ; 95 \% \mathrm{Cl}, 0.15-0.61)$ with an $\mathrm{RR}$ of $66 \%$, but did not in patients with PD-L1 $<50 \%$ (7). The two phase 3 trials are ongoing; the SKYSCRAPER-01 trial is exploring atezolizumab with or without tiragolumab in patients with previously untreated advanced or metastatic PD-L1-selected NSCLC; and the SKYSCRAPER-02 trial is exploring atezolizumab plus carboplatin or etoposide with or without tiragolumab in patients with untreated extensive-stage SCLC.

Vibostolimab (MK-7684) also showed good tolerability with or without pembrolizumab, anti-PD-1 antibody and preliminary efficacy in a phase I trial. The ORR was $7 \%$ with vibostolimab monotherapy and $5 \%$ with a vibostolimab/pembrolizumab combination, whereas the corresponding median duration of response was reportedly 9 months and 13 months, respectively (8). In part B of the trial for anti-PD-1/PD-L1 therapy-naïve patients, the vibostolimab/pembrolizumab combination demonstrated an ORR of $29 \%$ and a median PFS of 5.4 months. The ORR and median PFS were $46 \%$ and 8.4 months, respectively, in patients with PD-L1 $\geq 1 \%$, whereas that was $25 \%$ and 4.1 months, respectively, in patients with PD-L1 $<1 \%(9)$.

Other anti-TIGIT antibodies are under clinical investigation, including BMS-986207, ASP8374, AB154, BGB-A1217, and OMP313M32. Many new agents, such as COM902, IBI939, and EOS884448, are also going into early clinical development or are in preclinical development.

\section{Lymphocyte activation gene-3 (LAG-3)}

LAG-3/CD223 is a surface molecule located close to CD4 but sharing less than $20 \%$ homology. Like CD4, LAG-3 binds to a major histocompatibility complex-II (MHC-II) but with much stronger affinity, prohibits the binding of the same MHC molecule to T-cell receptor (TCR) and CD4, and thus directly hinders TCR signaling in the immune response. However, since its genetic or pharmacological deficiency makes it ineffective alone, it requires cooperation with other signals, such as blockade of the PD-1/PD-L1 pathway. In addition, LAG-3/MHC-II interaction may act as bi-directional inhibitory signaling shared by tumor cells and immune cells. Actually, aberrant LAG-3 expression is observed in many tumor types.

Relatlimab (BMS-986016) showed its efficacy with nivolumab in malignant melanoma patients who had already been exposed to anti-PD-1/PD-L1 therapy. The combination resulted in an ORR of $11.5 \%$ including one complete response (CR) and DCR of $49 \%$. An exploratory analysis in patients with LAG-3 $\geq 1 \%$ showed an RR of $18 \%$ and DCR of $64 \%$. Of note, RRs differed according to PD-L1 expression; for PD-L1 $\geq$ $1 \%$, the RR was $6.3 \%$; but for PD-L1 $<1 \%$, that was $27 \%$. For LAG-3 $<1 \%$, PD-L1 expression did not influence responses. For those who had been exposed to anti-CTLA-4 therapy, the RR was $24 \%$ when LAG-3 was $\geq 1 \%$, whereas that was $8.3 \%$ when LAG-3 $<1 \%$ (10).

Eftilagimod alpha (IMP321) is a soluble LAG-3 protein that binds MHC-II molecules to mediate antigen-presenting cells (APCs) and then activate CD-8 + T-cell, whereas anti-LAG-3 antibodies inhibit LAG-3 on T cells to restore T-cell activity. Combined with pembrolizumab, the ORR was $53 \%$ as first-line therapy for NSCLC patients and $39 \%$ as second-line therapy for immunotherapy-naïve head and neck squamous-cell carcinoma (HNSCC) patients (11).

Leramilimab (LAG525 or IMP701), when combined with spartalizumab (PDR001), an anti-PD-1 antibody, led to enduring responses in a phase I trial, including 2 of 8 mesothelioma patients and 2 of 5 triple-negative breast-cancer (TNBC) patients (12).

MK-4280 monotherapy demonstrated an RR of $6 \%$ and DCR of $17 \%$ in patients with advanced solid tumors, whereas an MK-4280/pembrolizumab combination had an RR of $27 \%$ and DCR of $40 \%$ (13). Of note, a biomarker-directed randomized phase II (MK-3475-95/KEYNOTE-495) study of pembrolizumab in combination with MK-1308 (anti-CTLA4), MK-4208 or lenvatinib for treatment-naïve NSCLC patients is ongoing. In the study, participants will be defined by gene-expression profiles and tumor-mutation burden before randomization.

In addition to classic IgG4 anti-LAG-3 antibodies, including relatilimab, leramilimab, MK-4280, fianlimab (REGN3767), TSR033, and BI-754111, different types of anti-LAG-3 antibodies are also under development. Sym022 is an Fc-inert human antibody and INCAGN02385 is an Fc-engineered IgG1k antibody. Of more interest, bi-specific antibodies targeting both LAG-3 and PD-1 were explored based on preclinical findings that both are highly upregulated on dysfunctional TILs and thus co-blockade demonstrates synergistic improvement of antitumor responses. Bi-specific antibodies are under clinical investigation, including tebotelimab (MGD013) and RO7247669. Tebotelimab is being explored not only as monotherapy but also in combination with either brivarinib, VEGFR2 inhibitor, or margetuximab, an anti-HER2 antibody.

\section{Transmembrane immunoglobulin and mucin domain 3 (TIM-3)}

TIM-3 is a glycoprotein with poorly understood signaling mediators and heterogeneous ligands. TIM-3 is often co-expressed with PD-1 in exhausted CD8 + cells in tumors that exhibit defects in proliferation and cytokine production. In addition, TIM-3+ and FoxP3 + Tregs seem to be a significant common feature across many tumor types. These cells are more suppressive than are TIM-3- and FoxP3 + Tregs owing to increased production of IL-10, and so promote dysfunctional CD8 + TILs. In preclinical models, its blockade synergizes with blockade of PD-1 
despite its lack of efficacy as a single agent.

Cobolimab (TSR022) was evaluated as monotherapy or in combination with TSR-042, an anti-PD-1 antibody. The benefit was observed in combination, with an ORR of $15 \%$ and DCR of $40 \%$ (14). Cobolimab is also being investigated in combination with either TSR-042 and TSR-033, anti-LAG-3 antibody, or TSR-042 and docetaxel.

Like anti-LAG-3 antibodies, many agents are under clinical investigation, including MBG453, Sym023, INCAGN2390, LY 3321367, BMS-986258 and SHR1702. A bispecific antibody, RO712661, targeting both TIM-3 and PD-1, also entered into clinical investigation.

\section{V-domain Ig suppressor of T-cell activation (VISTA or B7-H5 or PD-1 homolog)}

VISTA is highly expressed on mature APCs and, to a lesser extent, on Tregs and TILs. In addition, VISTA expression on infiltration myeloid cells is also consistently observed in many tumor types and increases with tumor progression, meaning that VISTA is active in regulating T-cell and myeloid functions in the tumor microenvironment (TME) $(15,16)$. Releasing suppressive activity of myeloid cells by the blockade of VISTA could synergize blockade of other IC inhibitors. In fact, the synergic effect of dual VISTA/PD-L1blockage was observed in animal models.

Anti-VISTA antibodies, such as JNJ-61610588 and HMBD-002, are under clinical investigation or about to enter clinical trials. Of interest, CA-170, an oral inhibitor targeting both PD-L1 and VISTA, showed preliminary efficacy. In a phase II trial, the clinical benefit rate (CBR) by irRECIST was $52 \%$ in treated Hodgkin lymphoma and more than $70 \%$ in treated NSCLC, but no objective response was observed $(17,18)$.

\section{B7-H3 (CD276)}

B7-H3 was initially discovered as a positive co-stimulator but has been found to be involved in the inhibition of $\mathrm{T}$ cells in recent studies (19). B7-H3 is expressed in the TME of many tumors and its blockade controlled tumor growth. The antitumor immunity was dependent on $\mathrm{CD}^{+} \mathrm{T}$ cells and NK cells (20).

Enoblituzumab (MGA271) has been studied in phase I trials, especially for B7-H3-expressing solid tumors. As monotherapy, enoblituzumab produced four partial responses (PRs) out of 54 patients with solid tumors. Of note, one PR was observed in 18 malignant melanoma patients who had already received either anti CTLA-4 or anti-PD-1 or both (21). With pembrolizumab, enoblituzumab resulted in an ORR of $35.7 \%$ for antiPD-1/PD-L1 therapy-naïve NSCLC and an ORR of $33.3 \%$ for anti-PD-1/PD-L1 therapy-naïve HNSCC, whereas RRs ranged from $0 \%$ to $7.7 \%$ for previously anti-PD-1/PD-L1 therapy-treated patients according to tumor types. Interestingly, high B7-H3 expression was related to better clinical activity; among 11 anti-PD1/PD-L1 therapy-naïve NSCLC patients with PD-L1 $<1 \%$ but $\mathrm{B} 7-\mathrm{H} 3 \geq 10 \%$, there were 5 PRs and 5 SDs, giving an RR of $45.5 \%$ and DCR of $90.9 \%$; and among 15 anti-PD1/PD-L1 therapy-naïve HNSCC patients with $\mathrm{B} 7-\mathrm{H} 3 \geq 10 \%, 6$ PRs and 5 SDs were observed, giving an RR of $40.0 \%$ and DCR of $73.3 \%$ (22).

Orlotamab (MGD009) is a bispecific antibody binding both CD3 on T cells and B7-H3 on tumor cells. However, it was placed on partial clinical hold because of hepatic adverse events. Omburtamab (8H9), an antibody drug conjugate labeled with radioactive iodine-131, is also being investigated in a phase II and III study for CNS tumors, including leptomeningeal metastases, based on clinical activity seen in two pivotal phase II studies.

\section{B- and T-lymphocyte attenuator (BTLA, CD272)}

BTLA is another inhibitory receptor that belongs to the CD28 immunoglobulin superfamily and is expressed on mature lymphocytes,-including Tregs, macrophages, and mature bone-marrow-derived DCs. Co-signaling molecules can be classified into two families based on their structure. One is the CD28 immunoglobulin superfamily, which includes co-stimulatory molecules, such as CD28, inducible co-stimulatory molecule (ICOS), and co-inhibitory molecules, such as CTLA-4, PD-1, TIM-3, LAG-3, TIGIT, and BTLA. The other is the tumor necrosis factor receptor superfamily (TNFRSF), which includes costimulatory molecules, such as CD27, CD30, 4-1BB, CD40, OX40 and GITR. Therefore, BTLA is similar to PD-1 and CTLA4, inhibiting T-cell activation and cytokine production. TAB004/ JSO04, the first anti-BTAL antibody, entered into clinical investigation.

\section{CO-STIMULATORY IMMUNE-CHECKPOINT AGONIST (Table 2)}

Co-stimulatory ICs are also the target of clinical development. Unlike IC inhibitors targeting co-inhibitory ICs, development of agonists is complicated for several reasons. One problem is the geometric constraints of the peculiar structure of receptors. The cross-linking properties, which are influenced by the interaction between targeting antibodies and $\mathrm{Fc} \gamma$ receptors on surrounding cells, may be a key for their efficacy, and therefore synthetic ligands could be more effective than are antibodies. A further complication is the contradictory role of co-stimulatory ICs on inducible effector T cells and on constitutively expressed regulatory $\mathrm{T}$ cells. The different expressions in T cells might allow designing dual activity agents that productively engage effector $T$ cells while selectively depleting regulatory $T$ cells, especially through antibody-dependent cellular cytotoxicity (ADCC). However, such agents might require sufficient density of ADCC-competent myeloid/NK cells or synergize by increasing innate infiltrates. They are also being investigated or developed as monotherapy or in combination with IC inhibitors, such as anti-CTLA4 or anti PD-1/PD-L1 antibodies. 
Table 2. Co-stimulatory immune checkpoint agonists

\begin{tabular}{|c|c|c|c|c|}
\hline Target & Agent & Company & $\begin{array}{l}\text { Clinical } \\
\text { phase }\end{array}$ & Findings \\
\hline \multirow[t]{4}{*}{ ICOS } & Vopratelimab (JTX-2011) & Jounce Therapeutics & $\mathrm{I} / \mathrm{II}$ & $\begin{array}{l}\text { - As monotherapy } \\
\text { - For ICOS high: ORR } 22.2 \%, \text { mPFS } 6.2 \text { mo \& mOS } 21.4 \text { mo } \\
\text { - For ICOS low: ORR 0\%, mPFS } 2 \text { mo \& mOS } 9 \text { mo } \\
\text { - } \pm \text { Nivolumab or ipimilumab }\end{array}$ \\
\hline & GSK3359609 & GlaxoSmithKline & III & $\begin{array}{l}\text { - For HNSCC, } \\
\text { - Onotherapy; ORR } 8 \% \\
\text { - GSK3359609/pembrolizumab; ORR 28\% }\end{array}$ \\
\hline & MEDI-570 & MedImmune & I & • T-cell lymphoma \\
\hline & KY1044 & Kymab Limited & $\mathrm{I} / \mathrm{II}$ & - \pm Atezolizumab \\
\hline \multirow[t]{9}{*}{$\begin{array}{l}\text { OX40 } \\
(\mathrm{CD} 134)\end{array}$} & PF-0451860 & Pfizer & II & $\begin{array}{l}\text { - Monotherapy; ORR } 4 \% \\
\text { - Avelumab } \pm \text { PF-0451860 or axitinib } \pm \text { PF-0451860 } \\
\text { - PF-0451860 } \pm \text { utomilumab (4-1BB agonist) }\end{array}$ \\
\hline & Tavolimab (MEDI-0562) & Medlmmune & I & - Monotherapy; 2 immune-related PR \\
\hline & MEDI6962 & MedImmune & $\mathrm{I} / \mathrm{II}$ & $\begin{array}{l}\text { - Monotherapy or MEDI6962/tremelimumab or } \\
\text { MEDI6962/ritiximab; ORR 0\% } \\
\text { - MEDI6962/durvalumab; ORR 5\% }\end{array}$ \\
\hline & GSK3174998 & GlaxoSmithKline & $\mathrm{I} / \mathrm{II}$ & $\begin{array}{l}\text { • Monotherapy: ORR 2.2\% } \\
\text { - GSK3174998/pembrolizumab; ORR 7.3\% }\end{array}$ \\
\hline & $\begin{array}{l}\text { Vonlerolizumab } \\
\text { (MOXR0916) }\end{array}$ & Genentech & I & - Vonlerolizumab/atezolizumab; ORR 4\% \\
\hline & BMS-986178 & Bristol-Myers Squibb & I/II & \\
\hline & INCAGN01949 & Incyte & $\mathrm{I} / \mathrm{II}$ & $\begin{array}{l}\text { • Monotherapy: ORR } 0 \% \\
\text { - + Nivolumab or ipilimumab or both }\end{array}$ \\
\hline & ABBV-368 & AbbVie & I & \\
\hline & ATOR-1015 & Alligator Bioscience & 1 & \\
\hline \multirow[t]{7}{*}{$\begin{array}{l}\text { GITR } \\
\text { (CD357) }\end{array}$} & TRX 518 & Leap Therapeutics, & $\mathrm{I} / \mathrm{II}$ & $\begin{array}{l}- \text { Monotherapy: ORR } 0 \% \\
\text { - } \pm \text { Nivolumab or pembrolizumab }\end{array}$ \\
\hline & MK-4166 & Merck Sharp \& Dohme & I & $\begin{array}{l}\text { - Monotherapy: ORR 0\% } \\
\text { - MK-4166/pembrolizumab, } \\
\text { - Escalation cohort; ORR 9\% } \\
\text { - Expansion cohort for melanoma } \\
\text { IC inhibitor naïve; ORR } 69 \% \\
\text { IC inhibitor treated; ORR 0\% }\end{array}$ \\
\hline & MK-1248 & $\begin{array}{l}\text { Merck Sharp \& Dohme } \\
\text { Corp }\end{array}$ & & $\begin{array}{l}\text { - Monotherapy; ORR 0\% } \\
\text { - MK-1248/pembrolizumab; ORR 18\% }\end{array}$ \\
\hline & BMS-986156 & Bristol-Myers Squibb & $\mathrm{I} / \mathrm{II}$ & $\begin{array}{l}\text { - Monotherapy: ORR 0\% } \\
\text { - BMS-986156/nivolumab } \\
\text { - HNSCC; ORR 14.3\% } \\
\text { - Cervical cancer; ORR 13.9\% } \\
\text { - Bladder cancer, ORR 10.7\% } \\
\text { - HCC, ORR 8.3\% } \\
\text { - NSCLC, ORR 2.7\% }\end{array}$ \\
\hline & INCAGN01876 & Incyte Corporation & I/II & $\begin{array}{l}\text { • } \pm \text { Pembrolizumab \& epacadostat } \\
\text { - + Nivolumab or ipilimumab or both }\end{array}$ \\
\hline & OMP-336B11 & $\begin{array}{l}\text { OncoMed } \\
\text { Pharmaceuticals }\end{array}$ & I & Terminated by sponsor decision \\
\hline & MEDI1873 & Medlmmune & 1 & • Monotherapy; ORR 0\% \\
\hline
\end{tabular}

\section{Inducible co-stimulator (ICOS, CD278)}

ICOS/CD278 is a disulfide-linked homodimer and a member of the B7/CD28 immunoglobulin superfamily that is expressed mainly on activated $T$ cells. It is structurally and functionally similar to CD28 Biologically, it is closely intertwined with CTLA-4 and therefore, it is required for and upregulated by CTLA-4 block- ade, meaning that it strongly synergizes with CTLA-4 blockade in poorly immunogenic tumors.

The first agent is vopratelimab (JTX-2011), which was designed to achieve dual agonist/Treg-depleting activity. The phase I/II ICONIC trial as monotherapy or combinational therapy with nivolumab for heavily treated patients did show that the emer- 
Table 2. Continued

\begin{tabular}{|c|c|c|c|c|}
\hline Target & Agent & Company & $\begin{array}{l}\text { Clinical } \\
\text { phase }\end{array}$ & Findings \\
\hline \multirow[t]{4}{*}{$\begin{array}{l}4-1 B B \\
(C D 137)\end{array}$} & Urelumab (BMS-663513) & Bristol-Myers Squibb & $\mathrm{I} / \mathrm{II}$ & $\begin{array}{l}\text { - Monotherapy for R/R NHL: } \\
\text { - DLBCL; ORR 6\% } \\
\text { - FL; ORR 12\% } \\
\text { - Other NHL; ORR 17\% } \\
\text { - Urelumab/rituximab: } \\
\text { - DLBCL; ORR 10\% } \\
\text { - FL; ORR 35\% } \\
\text { - Urelumab/nivolumab } \\
\text { - Melanoma; ORR 50\% } \\
\text { - NSCLC; ORR 5\% } \\
\text { - HNSCC, ORR 4.5\% }\end{array}$ \\
\hline & $\begin{array}{l}\text { Utomilumab } \\
\text { (PF-05082566) }\end{array}$ & Pfizer & $\mathrm{I} / \mathrm{II}$ & $\begin{array}{l}\text { - Monotherapy; ORR 3.8\% } \\
\text { - Merkel cell carcinoma; ORR } 13.4 \% \\
\text { - Utomilumab/rituximab for R/R NHL; ORR 21.2\% } \\
\text { - Utomilumab/pembrolizumab; ORR 26.1\% } \\
\text { - Utomilumab/mogamulizumab (CCR4 inhibitor); ORR 4.2\% }\end{array}$ \\
\hline & PRS-343 & Pieris Pharmaceuticals & I & - Monotherapy for heavily treated HER2 + patients; ORR $11 \%$ \\
\hline & INBRX-105 & Inhibrx & I & \\
\hline CD27 & Varlilumab (CDX-1127) & Celldex Therapeutics & $\mathrm{I} / \mathrm{II}$ & $\begin{array}{l}\text { - Monotherapy } \\
\text { - HD; ORR 10\% } \\
\text { - NHL; ORR 0\% }\end{array}$ \\
\hline CD70 & Cusatuzumab (ARGX-110) & Argenx & $\mathrm{I} / \mathrm{II}$ & $\begin{array}{l}\text { - Monotherapy for solid tumor; ORR 0\% } \\
\text { - Monotherapy for CTCL, ORR 23\% } \\
\text { - Cusatuzumab/azacitidine for AML, ORR } 92 \%\end{array}$ \\
\hline \multirow[t]{5}{*}{ CD40 } & CP-870893 & Pfizer & I & $\begin{array}{l}\text { - Monotherapy for solid tumor; ORR 0\% } \\
\text { - CP-870893/paclitaxel/carboplatin for solid tumors; ORR 20\% } \\
\text { - CP-870893/gemcitabine for pancreatic cancer; ORR 24\% } \\
\text { - CP-870893/pemetrexed/cisplatin for mesothelioma; ORR } \\
\quad 40 \%\end{array}$ \\
\hline & APX005M & Bristol-Myers Squibb & $\mathrm{I} / \mathrm{II}$ & $\begin{array}{l}\text { - APX005M/gemcitabine/nab-paclitaxel for pancreatic cancer; } \\
\text { ORR } 58 \%\end{array}$ \\
\hline & ChiLob7/4 & Cancer Research UK & I & \\
\hline & SEA-CD40 & Seattle Genetics & 1 & $\begin{array}{l}\text { • } \pm \text { Pembrolizumab or } \\
\text { pembrolizumab/gemcitabine/nab-paclitaxel }\end{array}$ \\
\hline & CDX-1140 & Celldex Therapeutics & I & - \pm Pembrolizumab or gemcitabine/nab-paclitaxel \\
\hline
\end{tabular}

AML: acute myelogenous leukemia, CCR4: C-C chemokine receptor 4, CTCL: cutaneous T-cell lymphoma, DLBCL: diffuse large B-cell lymphoma, GITR: glucocorticoid-induced TNFR-related, FL: follicular lymphoma, HCC: hepatocelluar carcinoma, HD: Hodgkin disease, HER2: human epidermal growth factor receptor 2, HNSCC: head and neck squamous cell carcinoma, ICOS: Inducible co-stimulator, NHL: non-Hodgkin lymphoma, NSCLC: Non-small cell lung cancer, mo: month, mOS: median overall survival, mPFS: median progression-free survival, ORR: overall response rate, RR: relapsed/refractory.

gence of ICOS-high CD4 + cells was associated with the ORR, PFS, and OS. Out of 18 ICOS-high patients, 4 showed responses, giving an ORR of $22.2 \%$ with median PFS and OS of 6.2 months and 21.4 months, respectively, whereas 32 ICOS-low patients did not show any response with median PFS and OS of 2 months and 9 months, respectively (23). Following a phase II EMERGY study of vopratelimab in combination with ipilimumab, an anti-CTLA4 antibody, in NSCLC and urothelial tumors, is now recruiting after failure of anti PD-1/PD-L1 therapy. A combination study with nivolumab or ipilimumab for advanced/ refractory solid tumors is also ongoing.

GSK33359609 is a pure agonist and is investigated with or without pembrolizumab in the INDUCE-1 study, in which an
ORR of $8 \%$ and $28 \%$ was observed in monotherapy and combination therapy, respectively, in an expansion cohort of previously treated HNSCC patients with median PFS of 5.6 months (24). MEDI-570, which has strong ADCC-mediated T-cell-depleting activity, is under investigation in T-cell lymphoma. KY1044 is a dual agonist/depleting activity and is under clinical investigation with or without atezolizumab, anti-PD-L1 antibody.

\section{OX40 (CD134 or TNFRSF4)}

OX40, GITR, and 4-1BB are structurally related as members of TNFRSF and inducible T-cell co-stimulators like ICOS. OX40 has important co-stimulatory functions during T-cell activation, mediating the survival and expansion of both $\mathrm{CD}^{+}$and $\mathrm{CD}^{+}$ 
T cells in cancer as well as in auto-immunity and infectious diseases. OX40 also involves in controlling effector and memory T-cell responses. So, targeting OX40-OX40L interaction can promote non-regulatory $\mathrm{CD}^{+}$and $\mathrm{CD}^{+}{ }^{+} \mathrm{T}$-cell survival, sustain anti-apoptotic protein expression like BCL-XL, BCL-2 and survivin, increase cytokine production like IL-2 and IFN- $\gamma$, boost tumor-specific effector T-cell responses, and augment tumor-specific memory T-cell generation following antigen challenge. Of interest, the application of an anti-OX40 agonist was shown to induce activation and proliferation of T-cell populations, but also resulted in upregulation of PD-L1 in tumors occurring between 2 and 3 weeks after the administration. These findings suggested synergism with anti-PD/PD-L1 inhibitors.

Most OX40-targeting agents were in development as monoclonal antibodies and their safety profiles were reportedly manageable in early-phase trials. They include PF04518600, GSK 3174998, MEDI0562, MEDI6469, BMS986178, INCAGN01949, and MOXR0916. Some of them showed preliminary but limited clinical activity. New agents such as KY-B602 are still under preclinical development. Less conventional molecules are also under clinical investigation. MEDI1109 is a bispecific antibody co-targeting OX40 and PD-L1, and ATOR-1015 is a bispecific antibody co-targeting OX40 and CTLA-4. The one has not entered into clinical development yet, but the latter has already entered early clinical investigation.

PF-04518600 showed only one PR out of 25 evaluable patients in a phase I trial (25). PF-04518600 is being explored in combination with either FDA-approved agents or new agents. For example, not only avelumab, an anti-PD-L1 inhibitor, or axitinib, a vascular endothelial growth factor receptor (VEGFR) tyrosine kinase inhibitor (TKI), with or without PF-04518600, is being investigated. PF-04518600 with or without utomilumab, an anit-4-1 BB agonist, is also being studied. However, disappointingly, PF-04518600 was not seen in the sponsor's pipeline.

GSK3174998 was studied with or without pembrolizumab. There was one PR out of 45 patients enrolled in the monotherapy part, and 2 CRs and 7 PRs out of 96 patients enrolled in the combination part (26). GSK3174998 is now being explored in combination with other agents, such as pembrolizumab or GSK1795091, a toll-like receptor 4 agonist (TLR4).

MEDI0562 as a single agent showed 2 immunity-related partial responses and $44 \%$ stable disease in 55 patients enrolled, $47 \%$ of whom had HNSCC (27). A study of MEDI0562 in combination with either durvalumab, anti-PD-L1, or tremelimumab, an anti-CTLA4, for advanced solid tumor completed the enrollment but the results are not reported yet.

MEDI6469 was also investigated but as monotherapy; there was no response (28). Like MEDI0562, MEDI6469 was also investigated with durvalumab, tremelimumb, or rituximab. However, only one PR was observed in MEDI6469/durvalumab combination, but no response was observed as a single agent or when combined with tremelimumab or ritixumab. Accordingly, the sponsor discontinued the development of the two agents.

Vonlerolizumab (MOXR0916) was evaluated with or without atezolizumab. A phase Ib dose-escalation study of MOXR0916 and atezolizumab showed 2 PRs out of 51 patients or an ORR of $4 \%$ (29). So, the sponsor discontinued the MOXR916 program.

INCAGN01949 did not induce any response in a phase I/II study (30). A study of a combination of INCAGN01949 with either nivolumab or ipilimumab or both has completed the enrollment.

\section{Glucocorticoid-induced TNFR-related (GITR, TNFRSF18)}

GITR is also known as activation-inducible tumor necrosis factor. It is constitutively and highly expressed in CD4+/CD25 + regulatory $\mathrm{T}$ cells but expressed inducibly on NK and effector $\mathrm{T}$ cells. On the other hand, its ligand (GITRL) is also expressed on non-lymphoid activated APCs and endothelial cells. Its role is complex and context-dependent. GITR-null T cells show increased proliferation upon TCR engagement and increased antigen-induced cell death. Conversely, the engagement of GITR through ligands or agonistic antibodies promotes the expansion of effector T cells and production of cytokines.

TRX518, a first IgG1 anti-GITR antibody, showed unremarkable toxicity but little efficacy as monotherapy (30). It reduced circulating and intratumoral Tregs and increased the $T_{\text {eff }} / T_{\text {reg }}$ ratio. In preclinical models using TRX518, GITR agonism was not sufficient to activate cytolytic T cells, because of persistent exhaustion, but combination with PD-L1 blockade reinvigorated T cells, suggesting the possibility of overcoming resistance (31).

In fact, as single anti-GITR antibodies, TRX518, MK-4166, MK-1248, BMS986156, and MEDI1837 demonstrated a manageable safety profile but disappointingly limited clinical activity or no objective response. When combined with anti-PD-1/PD-L1 antibody, preliminary clinical activity was observed without dose-limiting toxicities (DLTs).

MK4166, humanized IgG1 antibody, in combination with pembrolizumab, showed 4 PRs out of 45 patients or an ORR of $9 \%$ in a dose-escalation cohort. There were 4 CRs and 5 PRs or an ORR of $69 \%$ in 13 IC inhibitor-naïve melanoma patients in an expansion cohort, whereas there was no response in previously IC-inhibitor-treated patients (32).

MK1248, a humanized IgG4 antibody, in combination with pembrolizumab, resulted in $1 \mathrm{CR}$ and 2 PRs out of 17 patients, giving an ORR of $18 \%$, whereas there was no response with monotherapy (33).

BMS-986156 when combined with nivolumab showed some clinical activity with ORRs of $0 \%$ to $11.1 \%$ across the different dose cohorts. Of note, responses were observed in different tumor types with ORRs of $14.3 \%$ for HNSCC, $13.9 \%$ for cervical cancer, $10.7 \%$ for bladder cancer, $8.3 \%$ for hepatocellular carcinoma (HCC), and $2.7 \%$ for NSCLC (34). INCAGN01876 was studied alone or with pembrolizumab and epacadostat, and is now being studied with either nivolumab or ipilimumab or both. 
However, AMG228 was not under further development, because of a business decision based on a phase I study in which there was no evidence of T-cell activation or anti-tumor activity with AMG 228 monotherapy (35).

Besides antibodies, other agents, including OMP-336B11, a synthetic GITR ligands-FC fusion protein, and MEDI1873, a homogenous hexameric GITRL fusion protein, were investigated. However, further clinical development of OMP-336B11 and MEDI8373 is not planned, because of the sponsor's decision or lack of anti-tumor activity, respectively $(36,37)$.

\section{4-1BB (CD137 or TNFRSF9)}

4-1BB provides CD28-independent co-stimulation that can overcome anergy, since 4-1BB can play a role of cytokine induction, prevent activation-induced cell death, and upregulate cytotoxic T-cell activity, but also may also be involved in reduction of the infiltration of Tregs into tumors. Agonists can eradicate established tumors even in monotherapy or in combination with anti-PD-1, inducing memory CD8-mediated long-term rejection of poorly immunogenic tumors. Two agonistic antibodies have been studied well to date.

The clinical development of IgG4 urelumab (BMS-663513) has been held up transiently because of the occurrence of target dose-dependent and fatal liver toxicity, but was restarted after lowering doses as monotherapy and combination therapy (38). The toxicity is related to the S100A4 protein secreted by tumors and stromal cells and thus close monitoring of liver enzymes is needed (39). A preliminary clinical activity was observed in lymphoma patients. With urelumab monotherapy who were refractory or resistant to rituximab. The ORR was $6 \%$ in patients with DLBCL, $12 \%$ in follicular lymphoma $(\mathrm{FL})$, and $17 \%$ in other types of non-Hodgkin's lymphoma (NHL). With a urelumab and rituximab combination, the ORR was $10 \%$ for DLBCL and $35 \%$ for FL (40). In another study of combination with nivolumab, encouraging efficacy was observed in melanoma patients, with RR of $50 \%$. However, in NSCLC and HNSCC patients, clinical activity was disappointingly limited, with RRs of $5 \%$ and $4.5 \%$, although no significant added toxicity was observed (41).

The other IgG2 utomilumab (PF-05082566) did show a better safety profile with no dose-limiting toxicities (DLTs). It also showed preliminary clinical activity, in that ORR was 3.8\% for solid tumors and $13.3 \%$ for Merkel cell carcinoma with a complete response (42). With rituximab for refractory and resistant $\mathrm{NHL}$, it showed an ORR of $21.2 \%$, including 4 CRs and 7 PRs (43). Many combinations of utomilumab with other agents were also explored. The combination with pembrolizumab showed a favorable safety profile and preliminary clinical activity with an ORR of $26.1 \%$ (44). The combination with mogamulizumab, an anti-C-C chemokine receptor 4 (CCR4) antibody, showed a favorable safety profile but limited clinical activity, with an ORR of $4.2 \%$ (45).

In fact, urelumab caused dose-dependent hepatitis, limiting the dose, whereas utomilumab had a better safety profile but lower agonistic potency. To overcome these limitations, different approaches are being investigated, such as Fc-free tumor-targeted 4-1BB-agonistic trimer body, or bispecific antibodies cotargeting 4-1 BB and HER2 or PD-1.

$1 \mathrm{D} 8 \mathrm{~N} / \mathrm{C}$ EGa1 is a 4-1BB-agnostic trimeric antibody. Trimeric antibodies have two major advantages compared to conventional IgG monoclonal antibodies; one is they lack the fragment crystallizable $(\mathrm{Fc})$ region involved in 4-1BB-mediated toxicity; and the other is that they are trimeric or more physiological 4-1BBL. In a preclinical study, 1D8 N/C EGa1 did induce potent anti-tumor activity without systemic toxicity (46). However, it has not entered into clinical investigation yet.

PRS-343 co-targets 4-1BB and HER2 and showed a preliminary but promising RR of $11 \%$. Out of 18 evaluable but heavily treated HER2 + patients, 2 PRs were observed (47). However, PRS-343 trials are now suspended because of a partial clinical hold. INBRX-105 co-targets both 4-1BB and PD-1 and is now recruiting.

\section{CD27 (TNFRSF7) and CD70}

CD27 is another member of TNFRSF and a costimulatory molecule on T cells, mediating cellular activation, proliferation, effector function, and cell survival on binding to its unique ligand, CD70. Expression of CD70 is tightly controlled and upregulated exclusively upon activation on $\mathrm{T}$ cells, B cells, and certain subsets of dendritic cells (DCs).

Varlilumab (CDX-1127), an anti-CD27 agonistic antibody, launched a first-in-human, dose-escalation, and expansion study for hematologic malignancies. The study showed a favorable safety profile with no DLTs. One out of $10 \mathrm{HD}$ patients experienced CR, but out of $18 \mathrm{NHL}$ patients, none experienced any response (48).

Cusatuzumab (ARGX-110), an anti-CD70 antibody, was also investigated in solid tumors and hematologic malignancies. In a phase I study for solid tumors, neither DLTs nor an antitumor response was observed (49). However, for heavily treated CD70 + cutaneous T-cell lymphoma patients, as monotherapy the ORR was $23 \%$ with $1 \mathrm{CR}$ and 5 PRs, whereas, combined with azacitidine for newly diagnosed AML patients, ORR was amazingly $92 \%$, including $9 \mathrm{CR} / \mathrm{CRi}$ and 1 morphologically leukemia-free statues $(50,51)$.

During the last decade, three antibody-drug conjugates targeting CD70, including vorsetuzumab mafodotin (SGN-75), MDX-1203 (BMS-936561) and SGN-CD70A, reached clinical development for lymphoma but now all have been discontinued.

\section{CD40 (TNFRSF5)}

CD40 is expressed on a wide range of hematopoietic and nonhematopoietic cells, but its ligand, CD154 or CD40L, is restrictedly expressed on activated T cells, B cells and platelets. The activation of CD40 leads to upregulation of MHC molecules, immunoglobulin superfamily co-stimulatory molecules, such as CD86, and other TNFRSF ligands, such as CD137, GITR, and OX40. 
Selicrelumab (CP-870893) produced an RR of $27 \%$ in advanced melanoma, whereas none of the 14 patients with other solid tumors responded. Interestingly, one patient experienced long-lasting remission for more than 15 years (52). However, in a subsequent trial, including malignant melanoma patients, there was no response in solid tumors (53). Following combinations with cytotoxic chemotherapy were studied. With carboplatin/paclitaxel, selicrelumab produced an ORR of $20 \%$ in advanced solid tumors; with gemcitabine for metastatic pancreatic cancer, it produced an ORR of $24 \%$, and with cisplatin/ pemetrexed for malignant pleural mesothelioma, it resulted in an ORR of $40 \%$ (54-56).

Other agonistic anti-40 agonists, including APX005M, ChiLob 7/4, SEA-CD40, and CDX-1140, did not result in meaningful clinical activity (57-60). Recently, for newly diagnosed metastatic pancreatic cancer patients, a result of a phase $\mathrm{lb}$ trial of APX005M and chemotherapy with or without nivolumab reported an ORR of 58\% (61). A randomized phase II study of gemcitabine/nab-paclitaxel with or without APX005M (0.3 mg/ $\mathrm{kg}$ ) and with or without nivolumab is under way. Other antiCD40 agonists are also being investigated with pembrolizumab or gemcitabine/nab-paclitaxel or both.

\section{OTHER IMMUNOMODULATORS OR MEDIATORS} (Table 3)

\section{Adenosine pathway}

Adenosine (ADO) is an effective endogenous immunosuppressant in cancer as well as in normal condition. It is excreted by stressed or injured cells or is generated through dephosphorylation of adenosine-monophosphate by the enzyme CD73, ecto5 '-nucelotidase. Hypoxia and TGF- $\beta$ are key determinants of producing adenosine derivatives. Hypoxia induces release of ATP or NAD+, a possible source of bioactive adenosine, through the activity of multiple enzymes expressed on cancer cells, such as CD39 or CD73. ADO acts by binding the amine receptors expressed on immunity cells, especially $\mathrm{T}$ and NK cells, of which the CAMP-increasing adenosine receptor $2 \mathrm{a}$ and $2 \mathrm{~b}$, $\mathrm{A} 2 \mathrm{aR}$ and $\mathrm{A} 2 \mathrm{bR}$, play a key role. As a result, $\mathrm{ADO}$ provokes Tregs and myeloid-derived suppressor cells (MDSCs) accumulation, $\mathrm{T}_{\text {eff }}$ and NK inhibition, or cancer-associated fibroblast proliferation, finally generating tumorigenic TME. In fact, ADO and CD73 are widely expressed on a variety of cells in the TME. Therefore, inhibition of either CD73 or A2aR or both could improve the activity of cytotoxic lymphocytes and reduce tumor growth. Synergy with anti-PD1 or anti-PDL1 and anti CTLA-4 therapies was also observed in preclinical studies. Agents targeting CD73, such as oleclumab or BMS-986179, are under clinical development alone or with anti-PD1/PD-L1 or anti-CTLA4 antibody.

A phase I first-in-human study with oleclumab (MEDI9447) alone or in combination with durvalumab for patients with advanced pancreatic or microsatellite-stability colorectal cancer (CRC) showed a safety profile as manageable as that of mono- therapy, but showed a preliminary clinical activity in the combination arm only like that of many other new immunotherapeutic agents. There was 1 PR out of 21 CRC patients, giving an RR of $4.7 \%$, and 2 PRs out of 20 pancreatic-cancer patients, giving an RR of $5 \%$ (62).

A phase I study of BMS-986179 in which patients received 2-week BMS-986179 monotherapy, followed by the combination with nivolumab, also showed a finding similar to that of oleclumab, in that a single BMS-986179 therapy demonstrated complete and persistent CD73 target engagement in the tumor and periphery, and then the combination produced a PR in 7 out of 52 patients (63).

CPI-006, an anti-CD73 antibody, alone or in combination with pembrolizumab or ciforadenant (CPI-444), an A2aR antagonist, is under clinical investigation. AK119, an anti-CD73 antibody recently entered clinical investigation with or without AK104, a PD-1/CTLA-4 bispecific antibody. LY3475070, a CD73 oral inhibitor, is also being explored as monotherapy or combinational therapy with pembrolizumab. GS-1423, an anti-CD73TGF $\beta$-Trap bifunctional antibody, is being clinically investigated with or without chemotherapy.

On the other hand, small molecules targeting A2aR were also developed to competitively inhibit ADO binding. Ciforadenant (CPI-444), originally investigated for Parkinson's disease, was tested alone or with atezolizumab and showed a good safety profile as a single agent or in combinational therapy. However, the clinical activity was relatively disappointing. For previously treated RCC patients, ciforadenant alone resulted in an RR of $3 \%$ with a median PFS of 4.1 months, whereas a ciforadenant/ atezolizumab combination resulted in an RR of $11 \%$ with a median PFS of 5.8 months (64). For 18 NSCLC patient, only one PR was observed (65). Another study of ciforadenant with or without atezolizumab for heavily treated metastatic castrationresistant prostate cancer (CRPC) did not produce any objective response (66).

A phase I study of NIR178 as a single agent showed a favorable safety profile and clinical benefits in heavily treated NSCLC patients, with an RR of $11.8 \%$ including 1 CR (67). A subsequent phase I/II study of NIR178 and spartalizumab (PDR001), an anti-PD-1 antibody, showed clinical benefits in 2 patients including $1 \mathrm{CR}$, giving an RR of $8 \%$ (68). Following phase II studies in combination with spartalizumab are ongoing.

AZD4635 also showed clinical activity with manageable toxicities in a phase la study. Interestingly, at first 3 PRs were observed in 8 evaluable metastatic CRPC patients, including 1 PR in monotherapy and $1 \mathrm{CR}$ and $1 \mathrm{PR}$ in combination with durvalumab (69). More data with more metastatic CRPC patients were reported; in the monotherapy group, the ORR was $6.1 \%$, and in the combination group, it was $16.2 \%$, interestingly including 2 CRs. Of note, patients with a high adenosine gene signature in the peripheral blood showed longer PFS than did those with a low signature (median PFS 21 weeks vs 8.7 weeks; HR 0.5; $95 \mathrm{Cl}, 0.3-0.9$ ) (70). A following phase II study for $\mathrm{mCRPC}$ patients is ongoing. 
Update in cancer immunotherapy

Dae Ho Lee

Table 3. Other immunomodulators or mediators

\begin{tabular}{|c|c|c|c|c|}
\hline Target & Agent & Company & $\begin{array}{l}\text { Clinical } \\
\text { phase }\end{array}$ & Findings \\
\hline \multirow[t]{5}{*}{ CD73 } & Oleclumab (MEDI9447) & AstraZeneca & $\mathrm{l} / \mathrm{II}$ & $\begin{array}{l}\text { - Monotherapy for solid tumors; ORR 0\% } \\
\text { - Oleclumab/durvalumab, } \\
\text { - CRC; ORR } 4.7 \% \\
\text { - Pancreatic cancer; ORR } 5 \%\end{array}$ \\
\hline & BMS-986179 & Bristol-Myers Squibb & I & - BMS-986179/nivolumab; ORR 13.4\% \\
\hline & CPI-006 & Corvus Pharmaceuticals & I & \\
\hline & AK-119 & Akeso & I & $\bullet \pm$ AK104 (PD-1/CTLA-4 bispecific antibody) \\
\hline & LY3475070 & Eli Lilly & I & \\
\hline \multirow[t]{3}{*}{$\mathrm{A} 2 \mathrm{aR}$} & Ciforadenant (CPI-444) & Corvus Pharmaceuticals & I & $\begin{array}{l}\text { - For previously treated RCC, } \\
\text { - Monotherapy; ORR 3\% \& mPFS } 4.1 \mathrm{mo} \\
\text { - Ciforadenant/atezolizumab; ORR } 11 \% \text { \& mPFS of } 5.8 \text { mo } \\
\text { - For previously treated NSCLC; ORR 5.5\% } \\
\text { - For previously treated CRPC; ORR } 0 \%\end{array}$ \\
\hline & NIR178 & Novartis & $\mathrm{I} / \mathrm{II}$ & $\begin{array}{l}\text { - For previously treated NSCLC, } \\
\text { - Monotherapy; ORR } 11.8 \% \\
\text { - NIR178/spartalizumab; ORR } 8 \%\end{array}$ \\
\hline & AZD4635 & AstraZeneca & $\mathrm{l} / \mathrm{II}$ & $\begin{array}{l}\text { - For metastatic CRPC, } \\
\text { - Monotherapy; ORR 6.1\% } \\
\text { - AZD4635/durvalumab; ORR 16.2\% }\end{array}$ \\
\hline $\mathrm{A} 2 \mathrm{bR}$ & PBF-1129 & Palobiofarma SL & I & \\
\hline $\begin{array}{l}\mathrm{A} 2 \mathrm{aR} \& \\
\mathrm{~A} 2 \mathrm{bR}\end{array}$ & AB928 & Arcus Biosciences & I & \\
\hline \multirow[t]{2}{*}{ IDO-1,2 } & Indoximod & New Link Genetics & II & $\begin{array}{l}\text { - Sipuleucel-T } \pm \text { indoximod for refractory metastatic } \\
\text { prostate cancer, mPFS } 10.3 \text { mo vs } 4.1 \text { mo } \\
\text { - Indoximod/gemcitabine/nab-paclitaxel for pancreatic cancer, } \\
\text { ORR } 46.2 \% \\
\text { - Indoximod/pembrolizumab for melanoma, ORR } 53 \% \text { \& } \\
\text { mPFS } 12.4 \text { mo }\end{array}$ \\
\hline & Epacadostat & Incyte Biosciences & III & $\begin{array}{l}\text { - Pembrolizumab } \pm \text { epacadostat for melanoma, mPFS } 4.7 \text { mo } \\
\text { vs } 4.9 \text { mo }(\mathrm{HR} 1.00,95 \% \mathrm{Cl} 0.83-1.21 \text {; one-sided } \mathrm{P}=0.52) \\
\text { \& OS (HR 1.13,95\% Cl, 0.86-1.49; one-sided } \mathrm{P}=0.81)\end{array}$ \\
\hline \multirow{5}{*}{$\begin{array}{l}\text { CSF-1/CSF } \\
-1 \mathrm{R}\end{array}$} & Emactuzumab (RG7155) & Roche & $\mathrm{l} / \mathrm{II}$ & • Emactuzumab/paclitaxel; ORR 7\% \\
\hline & Lacnotuzumab (MCS110) & Novartis & 1 & - Lacnotuzumab/spartalizumab; ORR 2\% \\
\hline & PD-0360324 & Pfizer & I & \\
\hline & BMS-986227 & Bristol-Myers Squibb & I & \\
\hline & IMC-CS4 & Eli Lilly & I & \\
\hline \multirow[t]{7}{*}{ CD47 } & Hu5F9-G4 & Gilead Sciences & I & $\begin{array}{l}\text { - Hu5F9-G4/rituximab for rituximab-refractory NHL; ORR } \\
50 \% \\
\text { - Hu5F9-G4/azacitidine for chemotherapy-ineligible untreated } \\
\text { AML or high risk MDS;ORR } 53 \% \\
\text { - + Rituximab, cetuximab, avelumab or atezolizumab }\end{array}$ \\
\hline & CC-90002 & Celgene & I & - CC-90002/rituximab; ORR 13\% \\
\hline & AK117 & Akeso & I & \\
\hline & IBI188 & Innovent Biologics & I & $\bullet \pm$ Rituximab \\
\hline & IBI322 & Innovent Biologics & I & \\
\hline & TTI-622 & Trillium Therapeutics & I & $\begin{array}{l}\bullet \pm \text { Rituximab, nivolumab or carfilzomib } \\
\text { (proteasome-inhibitor) }\end{array}$ \\
\hline & ALX-148 & ALX Oncology & $\mathrm{I} / \mathrm{II}$ & $\begin{array}{l}\text { - ALX-148/rituximab; ORR 35\% } \\
\text { - Indolent NHL, ORR } 50 \% \\
\text { - Aggressive NHL, ORR } 31 \% \\
\text { - ALX-148/pembrolizumab for HNSCC } \\
\text { - IC inhibitor naïve; ORR } 40 \% \\
\text { - IC inhibitor treated; ORR 0\% } \\
\text { - ALX-148/trastuzumab for HER2 + G/GEJ cancer; ORR 21\% }\end{array}$ \\
\hline
\end{tabular}


Table 3. Continued

\begin{tabular}{|c|c|c|c|c|}
\hline Target & Agent & Company & $\begin{array}{l}\text { Clinical } \\
\text { phase }\end{array}$ & Findings \\
\hline \multirow{10}{*}{$\begin{array}{l}\text { Chemokine- } \\
\text { che- } \\
\text { mokine } \\
\text { receptor } \\
\text { inhibitor }\end{array}$} & $\begin{array}{l}\text { PF-04136309 } \\
\text { (CCR2 inhibitor) }\end{array}$ & Pfizer & I & $\begin{array}{l}\text {-PF-04136309/FOLFIRINOX; ORR 48.5\% } \\
\text { - PF-04136309/gemcitabine/nab-paclitaxel; ORR 60\% }\end{array}$ \\
\hline & $\begin{array}{l}\text { BMS-813160 } \\
\text { (CCR2/CCR5 inhibitor) }\end{array}$ & Bristol-Myers Squibb & $1 / I I$ & - BMS-813160/nivolumab/gemcitabine/nab-paclitaxel \\
\hline & $\begin{array}{l}\text { Carlumab } \\
\text { (CNTO888, anti-CCL2), }\end{array}$ & $\begin{array}{l}\text { Centocor Research \& } \\
\text { Development }\end{array}$ & I & - Monotherapy for metastatic CRPC; ORR 0\% \\
\hline & $\begin{array}{l}\text { Mogamulizumab } \\
\text { (KW0761, anti-CCR4) }\end{array}$ & Kyowa Kirin & $1 / I I$ & $\begin{array}{l}\text { - Mogamulizumab/nivolumab, } \\
\text { - For HCC, ORR } 27 \% \\
\text { - For pancreatic cancer, ORR } 20 \% \\
\text { - Mogamulizumab/durvalumab or } \\
\text { mogamulizumab/tremelimumab; ORR } 5.3 \% \\
\text { - Mogamulizumab/utomilumab; ORR } 4.2 \%\end{array}$ \\
\hline & $\begin{array}{l}\text { Reparixin (DF1681Y, } \\
\text { CXCR1/CXCR2 inhibitor) }\end{array}$ & $\begin{array}{l}\text { Dompé } \\
\text { Farmaceutici S.p.A }\end{array}$ & II & - Reparixin/paclitaxel; ORR 29.6\% \\
\hline & $\begin{array}{l}\text { Navarixin (MK-7123, } \\
\text { CXCR2 inhibitor) }\end{array}$ & $\begin{array}{l}\text { Merck Sharp \& } \\
\text { Dohme Corp }\end{array}$ & II & \\
\hline & $\begin{array}{l}\text { AZD5069 } \\
\text { (oral CXCR2 inhibitor) }\end{array}$ & Astra Zeneca & II & - AZD5069/durvalumab for pancreatic cancer; ORR 5.6\% \\
\hline & $\begin{array}{l}\text { Plerixafor (AMD3100, } \\
\text { oral CXCR4 inhibitor) }\end{array}$ & $\begin{array}{l}\text { Merck Sharp \& } \\
\text { Dohme Corp }\end{array}$ & II & \\
\hline & $\begin{array}{r}\text { LY2510924, (CXCR4 } \\
\text { peptide antagonist) }\end{array}$ & Eli Lilly & II & - LY2510924/durvalumab for pancreatic cancer; ORR 0\% \\
\hline & $\begin{array}{l}\text { Balixafortide } \\
\text { (POL6326, CXCR4 } \\
\text { peptide antagonist) }\end{array}$ & Polyphor & III & $\begin{array}{l}\text { - Balixafortide/eribulin for HER- breast cancer, ORR 30\% } \\
\text { - Eribulin } \pm \text { balixafortide for HER- breast cancer }\end{array}$ \\
\hline \multirow[t]{3}{*}{ KIRs } & IPH2011 & Innate Pharma & I & • Monotherapy; ORR 0\% \\
\hline & $\begin{array}{l}\text { Lirilumab } \\
\quad(\text { IPH2102/BMS-986015) }\end{array}$ & Bristol-Myers Squibb & $1 / I I$ & $\begin{array}{l}\text { - Monotherapy; ORR 0\% } \\
\text { - Lirilumab/nivolumab for HNSCC; ORR 24\% } \\
\text { - + Nivolumab/ipilimumab }\end{array}$ \\
\hline & Lacutamab (IPH4102) & Innate Pharma & II & - Monotherapy for CTCL; ORR 36.4\% \\
\hline NKG2A & Monalizumab (IPH2201) & Innate Pharma & II & - Monalizumab/cetuximab for HNSCC; ORR 20\% \\
\hline \multirow{5}{*}{$\begin{array}{l}\text { STING } \\
\text { agonist }\end{array}$} & MK-1454 & Merck Sharp \& Dohme & I & - MK-1454/pembrolizumab; ORR 24\% \\
\hline & ADU-S100 (MIW815) & Aduro Biotech & I & $\begin{array}{l}\text { - MIW815/spartalizumab, ORR 3\% } \\
\text { - + Pembrolizumab or } \pm \text { ipilimumab }\end{array}$ \\
\hline & GSK3745417 & GlaxoSmithKline & I & $\bullet \pm$ Pembrolizumab \\
\hline & TAK-676 & Takeda & I & - \pm Pembrolizumab \\
\hline & E7766 & Eisai & I & \\
\hline
\end{tabular}

A2aR: adenosine receptor 2a, A2bR: adenosine receptor 2a, AML: acute myelogenous leukemia, CCL: C-C chemokine ligand, CCR2: C-C chemokine receptor 2, CCR4: C-C chemokine receptor 4, CCR5: C-C chemokine receptor 5, CRC: colorectal cancer, CRPC: castration resistant prostate cancer, CSF-1/CSF-1R: colony stimulating factor-1 and colony stimulating factor-1 receptor, CTCL-4: cytotoxic T-lymphocyte associate protein 4, CTCL: cutaneous T-cell lymphoma, CXCR1/CXCR2: CXC chemokine receptor 1/2, CXCR4: CXC chemokine receptor 4, HCC: hepatocelluar carcinoma, HER2: human epidermal growth factor receptor 2, HNSCC: head and neck squamous cell carcinoma, HR: hazard ratio, G/GEJ: gastric/gastroesophageal junction, IC: immune checkpoint, IDO-1,2: Indoleamine-2,3-dioxygenase 1 and 2, KIR: killer-cell immunoglobulin-like receptors, MDS: myelodysplastic syndrome, NHL: non-Hodgkin lymphoma, NSCLC: Non-small cell lung cancer, ORR: overall response rate, mo: month, OS: median overall survival, mPFS: median progression-free survival, RCC: renal cell carcinoma, STING: stimulator of interferon genes.

More A2aR inhibitors are under preclinical development. In addition, an A2aR inhibitor, PBF-1129, an A2bR antagonist, and $\mathrm{AB} 928$, a dual antagonist of the $\mathrm{A} 2 \mathrm{aR}$ and $\mathrm{A} 2 \mathrm{bR}$, are also under clinical investigation (71). 


\section{Indoleamine-2,3-dioxygenase 1 (IDO-1) pathway}

IDO1, a cytosolic heme-containing enzyme, involves in the catabolism of tryptophan, which was first shown to be critical for maternal-fetal immunity tolerance. IDO-1 inhibits activation of immune system by inhibition of mammalian target of rapamycin (mTOR) through tryptophan depletion, formation of kynurenine which promotes Tregs through the aryl hydrocarbon receptor, and non-enzymatic signaling through its ITIM domain homologous to TIGIT. Interestingly, IDO1 is induced by IFN- $\gamma$, and thus could mediate $s$ primary or acquired resistance to antiCTLA-4.

Indoximod showed preliminary but promising results in combination with sipuleucel-T in refractory metastatic prostate cancer, with an increase in PFS to 10.3 months vs 4.1 months and in combination with chemotherapy in pancreatic adenocarcinoma with a median OS of 10.9 months and an ORR of $46.2 \%(72,73)$. For melanoma patients, with pembrolizumab, indoximod produced an ORR of $53 \%$ with a rate of CR of $18 \%$ and a median PFS of 12.4 months (74). Actually, indoximod is not an IDO-1 inhibitor but more of a tryptophan mimetic, directly relieving mTOR inhibition on lymphocytes.

On the other hand, epacadostat, an IDO-1-selective hydroxyamidine, showed good efficacy in initial clinical trials. However, it did not work better in the larger phase III ECHO-301 trial, in which epacadostat and pembrolizumab had been compared with pembrolizumab alone in advanced melanoma patients, thereafter blocking or slowing down the further investigation of IDO-1 inhibitors (75). However, it is still not clear if IDO1 is an inherently ineffective target or if a more potent and stable compound is needed. In fact, patients who were previously treated with CTLA-4 inhibitor or BRAF inhibitor were included in the $\mathrm{ECHO} 312$ trial. Those prior therapies might increase the IDO1 level and compensatory IDO2 in TME, increase cytotoxic TIL and IFN- $\gamma$, and decrease the effect of the combination.

\section{CSF-1 (colony stimulating factor-1) pathway}

CSF-1R, a tyrosine kinase receptor of the platelet-derived growth factor receptor (PDGFR) family, has two structurally unrelated ligands, CSF-1 and IL-34. Its blockade modulates the recruitment and phenotype of tumor-associated macrophages (TAMs), significantly increasing the efficacy of CTLA-4, PD-1/PD-L1, or IDO-1 inhibition. Actually, in solid tumors, macrophages represent the main immune population, consisting of up to $50 \%$ of the tumor mass. Macrophage plasticity allows these innate immune cells to adopt their M1-M2 polarization axis. TAMs usually show M2 or pro-tumor and anti-inflammatory phenotypes, whereas the M1 phenotype is associated with antitumor and pro-inflammatory functions. T cells themselves can also secrete CSF-1 upon PD-1 blockade, inducing secondary resistance. Therefore, many agents are currently in development.

Emactuzumab (RG7155), an anti-CSF-1R antibody, has been extensively studied in the CSF-1R-expressing tenosynovial giantcell tumors and its well-known toxicity characterized by facial edema and connective-tissue autoimmunity, such as lupus erythematosus and dermohypodermitis (76). In a phase I trial as monotherapy or in combination with paclitaxel for solid tumors, the ORR of $7 \%$ was observed in combination therapy only (77).

Lacnotuzumab (MCS110), an anti-CSF-1 antibody, with spartalizumab produced an RR of $2 \%$, although the safety profile was manageable (78).

Not only anti-CSF-1 antibodies, such as PD-0360324, but also anti-CSF-1R antibodies, such as cabiralizumab (BMS-986227) and IMC-CS4, are under clinical investigation, but many trials of anti-CSF-1 or CSF-1R antibodies were withdrawn or terminated because of a lack of interest. Recently, a phase II trial of cabiralizumab in combination with nivolumab announced that the study had failed to show improved PFS in pancreatic cancer patients.

Nevertheless, CSF1R-specific kinase inhibitors, including pexidartinib/PLX3397, linifanib/ABT869, OSI-930, GW2580, and ARRY-382, are in preclinical or early clinical development.

\section{CD47-SIRP $\alpha$ signaling pathway}

CD47, integrin-associated protein (IAP), belongs to the immunoglobulin superfamily and is highly overexpressed on the surface of various types of solid tumor cells. The CD47-signal-regulatory protein $\alpha(\mathrm{SIRP} \alpha)$ complex initiates inhibitory signaling pathways, leading $s$ to the evasion of malignant cells from phagocytosis by macrophages. CD47 blockade stimulates the release of chemokines promoting the recruitment and activation of macrophage. The blockade also promotes the adaptive immunity response, such as induction of antigen-specific CD8 ${ }^{+}$ T-cell proliferation as well as macrophage phagocytosis, but reduces regulatory $T$ cells.

In a phase Ib study of Hu5F9-G4 for 22 rituximab-refractory $\mathrm{NHL}$ patients, an Hu5F9-G4 and rituximab combination resulted in an ORR of $50 \%$ with a CR rate of $36 \%$ (79). Another phase lb study of Hu5F9-G4 for patients with hematologic malignancy, with azacitidine, 53\% of chemotherapy-ineligible untreated AML or high-risk MDS patients had a CR/CRi with good tolerability (80). In a phase I study for patients with solid tumors, Hu5F9-G4 produced 2 PRs among 62 patients, all of whom had ovarian and fallopian-tube cancers with remission duration of 5.2 months and 9.2 months, respectively (81). Studies of combination with rituximab, cetuximab, or atezolizumab are ongoing.

CC-90002, an anti-CD47 antibody, showed an ORR of $13 \%$ when combined with rituximab (82). However, a study for relapsed/refractory $\mathrm{AML}$ and high-risk MDS was terminated, because preliminary monotherapy data did not show a profile sufficiently encouraging for further dose escalation/expansion.

Phase I studies of AK117, IB188, and IBI322 are ongoing. The first two agents are a classical anti-CD47 antibodies and the last is an anti-CD47/PD-L1 bispecific antibody. In addition, SIRP $\alpha$-Fc fusion proteins, such as TTI-622 and ALX148, block the interaction of CD47- SIRP $\alpha$ signaling and are under cli- 
nical investigation.

ALX148 with rituximab for NHL patients produced an ORR of $35 \%$ across all tumor histologies with an RR of $50 \%$ in indolent $\mathrm{NHL}$ and $31 \%$ in aggressive NHL (83). With pembrolizumab or trastuzumab, AXL148 showed an RR of $40 \%$ with a median PFS of 4.6 months for IC inhibitor-naïve HNSCC, but an RR of $0 \%$ with a median PFS of 2 months for IC-inhibitor-treated HNSCC, and an RR of $21 \%$ with a median PFS of 2.2 months for HER2 + gastric or gastroesophageal junction cancer (84).

\section{Chemokine and chemokine receptor antagonists}

Chemokines are small polypeptides produced by immune cells and belong to the cytokine superfamily. To date, more than 50 chemokines are identified and classified into CC, CXC, CX3C, and C subfamilies according to the number and location of $\mathrm{N}$-terminal cysteine molecules. More than 20 chemokine receptors are also identified and classified depending on their ligands. However, a chemokine can bind to multiple chemokine receptors, but also a receptor can bind to multiple chemokines. Therefore, their role depends on the specific receptors of target cells. For example, the CC chemokine receptor type 4 (CCR4) is expressed on Tregs and other circulating/ tumor-infiltrating T cells as well as on T-lymphoma cells. Binding of chemokines such as CCL17 or CCL22 to CCR4 promotes recruitment of immunosuppressive Tregs. Depletion of Tregs may decrease the suppression of anti-tumor immunity and synergize with PD-1 blockade. Actually, many chemokine or chemokine receptor blockers were investigated in preclinical cancer models or in auto-immune diseases. Some of them were or are being investigated in cancer patients.

PF-04136309, an oral CCR2 inhibitor, was investigated in combination with FOLFIRINOX chemotherapy for pancreatic cancer and showed an ORR of $48.5 \%$ compared to that of $25 \%$ with FOLFIRINOX alone (85). PF-04136309 with nab-paclitaxel and gemcitabine for borderline resectable and locally advanced pancreatic cancer also showed a preliminary clinical activity with an RR of $60 \%$ (86). However, the sponsor made a business-related decision to terminate the study.

BMS-813160, an oral CCR2/5 inhibitor, is being studied with nivolumab, gemcitabine, and nab-paclitaxel for borderline resectable and locally advanced pancreatic cancer.

Carlumab (CNTO888), an anti-CCL2 antibody, failed to show efficacy in metastatic CRPC, with an ORR of $0 \%$, perhaps because of ineffectiveness of CNTO 888 in reducing CCL2 serum level (87).

Mogamulizumab (KW0761) is an anti-CCR4 antibody and was approved first for cutaneous T-cell lymphoma based on improvement of outcomes in a phase III study in which mogamulizumab had a PFS superior to that of vorinostat (median PFS 7.7 months vs 3.1 months; HR 0.53; 95\% Cl, 0.41-0.69) with a manageable safety profile (88). When combined with nivolumab, it produced 4 PRs in 15 HCC patients, giving an ORR of $27 \%$, and 3 PRs in 15 pancreatic adenocarcinomas, giving an RR of $20 \%$. Of note, during the treatment, the decrease of effector Tregs (CD4 ${ }^{+}$CD45RA ${ }^{-}$FoxP $\left.{ }^{\text {high }}\right)$ and increase of $\mathrm{CD}^{+} \mathrm{T}$ cells in tumor-infiltrating lymphocytes was observed (89). However, with durvalumab or tremelimumab, it did not produce potent antitumor efficacy in solid tumors, giving an RR of only $5.3 \%$. With durvalumab, one patient with alveolar soft-part sarcoma reached a PR, whereas with tremelimumab, one patient with prostate cancer reached a PR (90).

Reparixin, an oral CXCR1/CXCR2 inhibitor, was studied with weekly paclitaxel and showed an ORR of $29.6 \%$ (91). Navarixin (MK-7123), an oral CXCR2 antagonist, is also under investigation with pembrolizumab. AZD5069, oral CXCR2 inhibitor, is being evaluated in a phase II study in combination with durvalumab for pancreatic cancer or with enzalutamide for CRPC.

Plerixafor (AMD3100), a CXCR4 antagonist, is approved for the mobilization of hematopoietic stem cells for transplantation but also is being studied in combination.

LY2510924, a CXCR4 peptide antagonist, has also been investigated with chemotherapy or sunitinib but failed to show clinical benefits $(92,93)$. Recently, a phase I study of combination with durvalumab reported no response, although it was a dose-finding study with a small sample (94).

Balixafortide (POL6326), a CXCR4 peptide antagonist, produced an RR of $30 \%$ when combined with eribulin for HER2 negative breast cancer and suggested a better survival outcome (95, 96). A phase III study of the combination is in progress.

\section{Agents targeting NK cells}

NK cells play a role at the intersection between the adaptive and the immune response. In solid tumors, NK-cell function is impaired significantly during tumor development and progression. Activating receptors of NK cells, such as NKG2D, CD226, and NKp30, are downregulated, whereas inhibitory receptors, such as killer-cell immunoglobulin-like receptors (KIRs) and NKG2A/CD94, are upregulated. Other inhibitory ICs, such as PD-1, TIM-3, LAG-3, and TIGIT, are also upregulated on NK cells. Therefore, the blockades of the ICs can reverse both NK-cell and T-cell function. However, immunotherapy targeting NK-cell checkpoints is being explored to prevent tumor escape from immune surveillance and to restore the anti-tumor capacity of NK cells.

KIRs belong to the Ig superfamily and interact with MHC-I molecules on target cells, comprising both activating and inhibitory receptors. The first-in-class IgG4 monoclonal antibody was IPH2101, which targets an epitope of KIR2D and thus blocks HLA-C-binding KIR2D receptors. A phase I trial of IPH2101 for relapsed or refractory multiple myeloma reported that it was safe and tolerable, but did not produce any objective response (97). Another phase I trial for AML patients in complete remission showed a favorable safety profile without DLTs and trends toward improvement of survival outcomes (98). Subsequently, lirilumab (IPH2102/BMS-986015), the second-generation anti-KIR antibody, was found to have a good safety profile but still had 
no responses in solid and hematologic malignancy, even though full KIR occupancy was sustained (99). Therefore, the sponsor decided to stop enrollment for myeloid malignancies and not pursue the development for the disease. With nivolumab, interim data of phase I/II of lirilumab for 29 HNSCC patients showed an ORR of $24 \%$ but might fail to a provide clear evidence of benefit (100). A phase I trial of combination with nivolumab and ipilimumab is open but not recruiting yet.

Lacutamab (IPH4102), a first-in-class anti-KIR3DL2 antibody, was shown to be safe and encouraging in clinical activity, with an ORR of $36.4 \%$ in a phase I clinical trial for relapsed or refractory cutaneous T-cell lymphoma (101). Phase II studies of IPH4102 alone or combined with chemotherapy are ongoing.

NKG2A (CD159) and CD94, a heterodimer inhibitory receptor of the C-type lectin family, recognizes a non-classical MHC-I molecule, HLA-E, as a ligand. Tumor cells upregulate HLA-E expression in order to avoid being killed by NK cells. Monalizumab (IPH2201, anti-NKG2A) has been explored in various tumors and shown to be well tolerated but has no responses. However, when combined with cetuximab, monalizumab produced an ORR of $20 \%$ in HNSCC patients who had been previously treated with platinum chemotherapy and anti-PD-1/ PD-L1 inhibitors (102). A following phase II study is ongoing.

\section{Stimulator of interferon genes (STING)}

The stimulator of interferon genes (STING) is a cytosolic protein and is activated by the enzyme cyclic-GMP-AMP synthase (cGAS). The cGAS-STING pathway is the central cellular cytosolic DNA sensor, allowing innate immunity to respond to infection, inflammation, and even cancer. The activation of the pathway after sensing cytosolic self or foreign DNA induced the production of type I interferons and aroused immune responses mediated by immune cells, including CD8 + T cells and NK cells. The pathway is also involved in cancer-cell senescence, meaning that the cGAS-STING signaling promotes the senescence via the secretion of chemokines, pro-inflammatory cytokines, growth factors, and proteases. The STING agonists, including ADU-S100 (MIW815), MK-1454, GSK3745417, and E7766, are under clinical investigations.

MK-1454 was shown to be safe as monotherapy and in clinical activity in combination with pembrolizumab with an ORR of $24 \%$ (103).

ADU-S100 (MIW815) in combination with spartalizumab showed 2 PRs out of 66 patients treated (104). The sponsor dropped MIW815 from its portfolio, but the developer continues the development of ADU-S100 in combination.

\section{CONCLUSION}

There are many immune-oncologic (IO) agents on clinical investigation as a single agent or in combination. More agents are also in preclinical development. Actually, more agents with different targets or therapeutics with different mechanisms are not covered in this review. For example, toll-like receptors, interleukin- 2 receptors, and arginase might be the targets. Cancer vaccines, oncolytic viruses, or even new CAR-T cells might be the therapeutics.

Nonetheless, many challenges are to be faced and should be overcome. As already seen in the agents mentioned above, a few agents showed promising clinical activity, but most showed no or minimal activity as monotherapy. Even the clinical activity of the combinations did not seem to be dramatically better than that of standard treatments, raising a concern whether the activity translates into a meaningful survival benefit. The first challenge might be the inability to accurately predict patient responses or to select appropriate patients because of lack of information on relevant biomarkers or surrogates. As the immune system is very dynamic and complex, identification of relevant biomarkers might be more difficult than in oncogene-driven personalized medicine. Even after the biomarkers are identified, if present, their role might change during the treatment, i.e., the spatio-temporal variation or the evolution of primary or acquired resistance.

The second issue relates to the paradox of choice. The investigation of new targets and pathways is very important in order to develop new therapeutics. However, whereas the pipeline of IO agents or therapeutics is ever-expanding, choosing the best or most appropriate treatment among many available options might become more difficult than before. The fact that we already have standard modalities to treat cancer patients other than immunotherapy, such as traditional cytotoxic chemotherapy or new oncogene-driven molecular targeted therapy, makes it much more complicated and complex. In fact, as more clinical trials of $\mathrm{IO}$ agents or treatments will be or are being conducted, accordingly enrolling subjects into each clinical becomes more difficult and problematic. Therefore, to find the best way to give immunotherapy concurrently or sequentially with not only standard treatment but also newly available treatment becomes more important than finding new targets and agents in a sense.

After all, the development of platforms not only to identify relevant biomarkers but also to give a comprehensive view or information should run parallel to the development of new therapeutics and discovery of new biomarkers. Despite many challenges and issues, immunotherapy is an important part of cancer treatment, giving us an opportunity of lengthening our life, improving our quality of life or even having a cancer-free life.

\section{CONFLICTS OF INTEREST}

I declare that I have received honoraria for consulting or lectures from Abbvie, AstraZeneca, Boehringer-Ingelheim, BristolMyers Squibb, ChongKunDang, CJ Healthcare, Eli Lilly, Green Cross, Genexine, GlaxoSmithKline, Janssen, Merck, MSD, Mundipharma, Novartis, Pfizer, Roche, Samyang Biopharm, ST Cube, and Takeda outside the submitted work. 


\section{REFERENCES}

1. Anderson AC, Joller N and Kuchroo VK (2016) Lag-3, Tim-3, and TIGIT co-inhibitory receptors with specialized functions in immunity regulation. Immunity 44, 989-1004

2. Koyama S, Akbay EA, Li YY et al (2016) Adaptive resistance to therapeutic PD-1 blockade is associated with upregulation of alternative immunity checkpoints. Nat Commun 7, 10501

3. Lepletier A, Madore J, O'Donnell JS et al (2020) Tumor CD155 expression is associated with resistance to antiPD1 immunotherapy in metastatic melanoma. Clin Cancer Res 26, 3671-3368

4. He W, Zhang H, Han F et al (2017) CD155T/TIGIT Signaling regulates $\mathrm{CD}^{+}{ }^{+} \mathrm{T}$-cell metabolism and promotes tumor progression in human gastric cancer. Cancer Res 77, 6375-6388

5. Chauvin J-M, Pagliano O, Fourcade J et al (2015) TIGIT and PD-1 impair tumor antigen-specific CD8 ${ }^{+}$T cells in melanoma patients. J Clin Invest 125, 2046-2058

6. Bendell JC, Bedard P, Bang YJ et al (2020) Phase la/lb dose-escalation study of the anti-TIGIT antibody tiragolumab as a single agent and in combination with atezolizumab in patients with advanced solid tumors. Clin Cancer Res 80 (suppl 16), abstract CT302

7. Rodriguez-Abreu D, Johnson ML, Hussein MA et al (2020). Primary analysis of a randomized, double-blind, phase II study of the anti-TIGIT antibody tiragolumab (tira) plus atezolizumab (atezo) versus placebo plus atezo as firstline (1L) treatment in patients with PD-L1-selected NSCLC (CITYSCAPE). J Clin Oncol 38 (suppl 15), abstract 9503

8. Ahn MJ, Niu J, Kim DW et al (2020) Vibostolimab, an anti-TIGIT antibody, as monotherapy and in combination with pembrolizumab in anti-PD-1/PD-L1-refractory NSCLC. Ann Oncol 31 (suppl 4), S887, abstract 1400P

9. Niu J, Nagrial A, Voskoboynik M et al (2020) Safety and efficacy of vibostolimab, an anti-TIGIT antibody, plus pembrolizumab in patients with anti-PD-1/PD-L1-naive NSCLC. Ann Oncol 31 (suppl 4), S891-892, abstract 1410P

10. Ascierto PA, Bono P, Bhatia S et al (2017) Efficacy of BMS-986016, a monoclonal antibody that targets lymphocyte activation gene-3 (LAG-3), in combination with nivolumab in pts with melanoma who progressed during prior anti-PD-1/PD-L1 therapy (mel prior IO) in all-comer and biomarker-enriched populations. Ann Oncol 28 (suppl 5), V611-612, abstract LBA18

11. Felip E, Doger B, Majem M et al (2020) Initial results from a phase II study (TACTI-002) in metastatic non-small cell lung or head and neck carcinoma patients receiving eftilagimod alpha (soluble LAG-3 protein) and pembrolizumab. J Clin Oncol 38 (suppl 15), abstract 3100

12. Hong DS, Schoffski P, Calvo A et al (2018) Phase I/II study of LAG525 \pm spartalizumab (PDR001) in patients (pts) with advanced malignancies. J Clin Oncol 36 (suppl 15), abstract 3012

13. Lakhani B, Bauer TM, Abraham AK et al (2018) The anti-LAG-3 antibody MK-4280 as monotherapy and in combination with pembrolizumab for advanced solid tumors: first-in-human phase 1 dose-finding study. J Immunother Cancer 6 (suppl 1), 115 abstract O26
14. Davar D, Boasberg P, Eroglu Z et al (2018) A phase 1 study of TSR-022, an anti-TIM-3 monoclonal antibody, in combination with TSR-042 (anti-PD-1) in patients with colorectal cancer and post-PD-1 NSCLC and melanoma. J Immunother Cancer 6 (suppl 1), 114 abstract O21

15. Villarroel-Espindola F, Yu X, Datar I et al (2018) Spatially resolved and quantitative analysis of VISTA/PD-1H as a novel immunotherapy target in human non-small cell lung cancer. Clin Cancer Res 24, 1562-1573

16. Deng J, Li J, Sarde A et al (2019) Hypoxia-induced VISTA promotes the suppressive function of myeloidderived suppressor cells in the tumor microenvironment. Cancer Immunol Res 7, 1019-1090

17. Radhakrishnan VS, Bakhshi S, Prabhash K et al (2018) Phase 2 trial of CA-170, a novel oral small molecule dual inhibitor of immunity checkpoints VISTA and PD-1, in patients (pts) with advanced solid tumor and Hodgkin lymphoma. J Immunother Cancer 6 (suppl 1), 115 abstract P714

18. Radhakrishnan V, Banavali S, Gupta S et al (2019) Excellent CBR and prolonged PFS in non-squamous NSCLC with oral CA-170, an inhibitor of VISTA and PD-L1. Ann Oncol 30 (suppl 5), V475-532 abstract 3012

19. Suh WK, Gajewska BU, Okada $\mathrm{H}$ et al (2003) The B7 family member B7-H3 preferentially down-regulates $T$ helper type 1-mediated immunity responses. Nat Immunol 4, 899-906

20. Lee Y, Martin-Orozco N, Zheng P et al (2017) Inhibition of the B7-H3 immunity-checkpoint limits tumor growth by enhancing cytotoxic lymphocyte function. Cell Res 27, 1034-1045

21. Powderly J, Cote G, Flaherty K et al (2015) Interim results of an ongoing phase 1, dose escalation study of MGA271 (Fc-optimized humanized anti-B7-H3 monoclonal antibody) in patients with refractory $\mathrm{B} 7-\mathrm{H} 3$-expressing neoplasms or neoplasms whose vasculature expresses B7-H3. J Immunother Cancer 3 (suppl 2), abstract O8

22. Aggarwal C, Joshua A, Ferris R et al (2018) A phase 1, open-label, dose escalation study of Enoblituzumab in combination with Pembrolizumab in patients with select solid tumors. J Immunotherapy Cancer 6 (suppl 2), 114, abstract P24

23. Yap TA, Gainor JF, Callahan MK et al (2019) Improved progression-free and overall survival (PFS/OS) in patients (pts) with emergence of JTX-2011 associated biomarker (ICOS high CD4 T cells) on the ICONIC trial. Cancer Res 79 (suppl 13), abstract CT189

24. Rischin D, Groenland SL, Lim AML et al (2019) Inducible $\mathrm{T}$ cell costimulatory (ICOS) receptor agonist, GSK 3359609 (GSK609) alone and in combination with Pembrolizumab (pembro): preliminary results from INDUCE-1 expansion cohorts (EC) in head and neck squamous cell carcinoma (HNSCC). Ann Oncol 30 (suppl 5), V449-474, abstract 4541

25. Diab A, El-Khoueiry A, Eskens FA et al (2016) A first-inhuman (FIH) study of PF-04518600 (PF-8600) OX40 agonist in adult patients (pts) with select advanced malignancies Ann Oncol 27 (suppl 6), V359-378, abstract 3148

26. Postel-Vinay S, Lam VK, Ros W et al (2020) Abstract CT150: A first-in-human phase I study of the OX40 ago- 
nist GSK3174998 (GSK998) +/ - pembrolizumab in patients (Pts) with selected advanced solid tumors (ENGAGE-1). Cancer Res 80 (suppl 16), abstract CT150

27. Glisson BS, Leidner RS, Ferris RL et al (2020) Safety and clinical activity of MEDI0562, a humanized OX40 agonist monoclonal antibody, in adult patients with advanced solid tumors. Clin Cancer Res 26, 5358-5367

28. Curti BD, Kovacsovics-Bankowski $\mathrm{M}$, Morris $\mathrm{N}$ et al (2013) OX40 is a potent immunity-stimulating target in late-stage cancer patients. Cancer Res 73, 7189-7198

29. Infante JR, Hansen AR, Pishvaian MJ et al (2016) A phase $\mathrm{lb}$ dose escalation study of the OX40 agonist MOXR0916 and the PD-L1 inhibitor atezolizumab in patients with advanced solid tumors. J Clin Oncol 34 (suppl 15), abstract 101

30. Koon H, Shepard D, Merghoub T et al (2016) First-inhuman phase 1 single-dose study of TRX-518, an antihuman glucocorticoid-induced tumor necrosis factor receptor (GITR) monoclonal antibody in adults with advanced solid tumors. J Clin Oncol 34 (suppl 15), abstract 3017

31. Zappasodi R, Sirard C, Li Y et al (2019) Rational design of anti-GITR-based combination immunotherapy. Nat Med 25, 759-766

32. Papadopoulos KP, Autio KA, Golan T et al (2019) Phase 1 study of MK-4166, an anti-human glucocorticoid-induced tumor necrosis factor receptor (GITR) antibody, as monotherapy or with pembrolizumab (pembro) in patients (pts) with advanced solid tumors. J Clin Oncol 37 (suppl 15), abstract 9509

33. Geva R, Voskoboynik M, Dobrenkov K et al (2020) Firstin-human phase 1 study of MK-1248, an anti-glucocorticoid-induced tumor necrosis factor receptor agonist monoclonal antibody, as monotherapy or with pembrolizumab in patients with advanced solid tumors. Cancer 15, 49264935

34. Heinhuis KM, Carlino M, Joerger M et al (2020) Safety, tolerability, and potential clinical activity of a glucocorticoid-induced TNF receptor-related protein agonist alone or in combination with nivolumab for patients with advanced solid tumors: a phase $1 / 2 \mathrm{a}$ dose-escalation and cohort-expansion clinical trial. JAMA Oncol 6, 100-107

35. Tran B, Carvajal RD, Marabelle A et al (2018) Dose escalation results from a first-in-human, phase 1 study of glucocorticoid-induced TNF receptor-related protein agonist AMG 228 in patients with advanced solid tumors. J Immunother Cancer 6, 93

36. Balmanoukian A, Infante JR, Aljumaily R et al (2020) Safety and clinical activity of MEDI1873, a novel GITR agonist, in advanced solid tumors. Clin Cancer Res 26, 6196-6203

37. Chan IH, Xie MH, Lam A et al (2018) In vitro functional activity of OMP-336B11, a GITRL-FC fusion protein, on primary human immunity cells. Cancer Res 78 (suppl 13), abstract 2726

38. Segal NH, Logan TF, Hodi FS et al (2017) Results from an integrated safety analysis of urelumab, an agonist antiCD137 monoclonal antibody. Clin Cancer Res 23, 19291936.

39. Zhang J, Song K, Wang J et al (2018) S100A4 blockage alleviates agonistic anti-CD137 antibody-induced liver pathology without disruption of antitumor immunity. OncoImmunology 7, e1296996

40. Timmerman J, Herbaux C, Ribrag V et al (2020) Urelumab alone or in combination with rituximab in patients with relapsed or refractory B-cell lymphoma. Am J Hematol 95, 510-520

41. Massarelli E, Segal NH, Ribrag V et al (2016) Clinical safety and efficacy assessment of the CD137 agonist urelumab alone and in combination with nivolumab in patients with hematologic and solid tumor malignancies. J Immunother Cancer 4, 82 abstract O7

42. Segal $\mathrm{NH}, \mathrm{He} A R$, Doi $\mathrm{T}$ et al (2018) Phase I study of single-agent Utomilumab (PF-05082566), a 4-1BB/CD137 agonist, in patients with advanced cancer. Clin Cancer Res 24, 1816-1823

43. Gopal AK, Levy R, Houot R et al (2020) First-in-human study of Utomilumab, a 4-1BB/CD137 agonist, in combination with Rituximab in patients with follicular and other $\mathrm{CD} 20^{+}$non-Hodgkin lymphomas. Clin Cancer Res 26, 2524-2534

44. Tolcher AW, Sznol M, Hu-Lieskovan S et al (2017) Phase Ib study of utomilumab (PF-05082566), a 4-1BB/CD137 agonist, in combination with pembrolizumab (MK-3475) in patients with advanced solid tumors. Clin Cancer Res 23, 5349-5357

45. Cohen EEW, Pishvaian MJ, Shepard DR et al (2019) A phase lb study of utomilumab (PF-05082566) in combination with mogamulizumab in patients with advanced solid tumors. J Immunother Cancer 7, 342

46. Compte M, Harwood SL, Muñoz IG et al (2018) A tumor-targeted trimeric 4-1BB-agonistic antibody induces potent anti-tumor immunity without systemic toxicity. Nat Commun 9, 4809

47. Piha-Paul S, Bendell J, Tolcher A et al (2019) Phase 1 dose escalation study of PRS-343, a HER2/4-1BB bispecific molecule, in patients with HER2 + malignancies. J Immunother Cancer 8 (suppl 1), abstract LBA O82

48. Ansell SM, Flinn I, Taylor MH et al (2020) Safety and activity of varlilumab, a novel and first-in-class agonist anti-CD27 antibody, for hematologic malignancies. Blood Adv 4, 1917-1926

49. Aftimos P, Rolfo C, Rottey S et al (2017) Phase I dose-escalation study of the anti-CD70 antibody ARGX110 in advanced malignancies. Clin Cancer Res 23, 64116420

50. Bagot M, Maerevoet M, Zinzani PL et al (2018) ARGX110 for treatment of CD70-positive advanced cutaneous T-cell lymphoma in a phase 1/2 Clinical Trial. Blood 132 (suppl 1), 1627

51. Ochsenbein AF, Riether $C$, Bacher $U$ et al (2018) ARGX110 targeting CD70, in combination with azacitidine, shows favorable safety profile and promising anti-leukemia activity in newly diagnosed AML patients in an ongoing phase 1/2 clinical trial. Blood 132 (suppl 1), abstract 2680

52. Vonderheide RH, Flaherty KT, Khalil M et al (2007) Clinical activity and immunity modulation in cancer patients treated with CP-870893, a novel CD40 agonist monoclonal antibody. J Clin Oncol 25, 876-883 
53. Ruter J, Antonia SJ, Burris HA $3^{\text {rd }}$ et al (2010) Immunity modulation with weekly dosing of an agonist CD40 antibody in a phase I study of patients with advanced solid tumors. Cancer Biol Ther 10, 983-993

54. Vonderheide RH, Burg JM, Mick R et al (2013) Phase I study of the CD40 agonist antibody CP-870893 combined with carboplatin and paclitaxel in patients with advanced solid tumors. Oncoimmunology 2, e23033

55. Beatty GL, Torigian DA, Chiorean EG et al (2013) A phase I study of an agonist CD40 monoclonal antibody (CP-870893) in combination with gemcitabine in patients with advanced pancreatic ductal adenocarcinoma. Clin Cancer Res 19, 6286-6295

56. Nowak AK, Cook AM, McDonnell AM et al (2015) A phase $1 \mathrm{~b}$ clinical trial of the CD40-activating antibody CP-870893 in combination with cisplatin and pemetrexed in malignant pleural mesothelioma. Ann Oncol 26, 24832490

57. Johnson M, Fakih $M$, Bendell J et al (2017) First in human study with the CD40 agonistic monoclonal antibody APX005M in subjects with solid tumors. J Immunother Cancer 5 (suppl 3), abstract 89

58. Vitale LA, Thomas LJ, He LZ et al (2019) Development of CDX-1140, an agonist CD40 antibody for cancer immunotherapy. Cancer Immunol Immunother 68, 233245

59. Grilley-Olson J, Curti BD, Smith DC et al (2018) SEACD40, a non-fucosylated CD40 agonist: interim results from a phase 1 study in advanced solid tumors. J Clin Oncol 36 (suppl 15), abstract 3093

60. Johnson $\mathrm{P}$, Challis R, Chowdhury F et al (2015) Clinical and biological effects of an agonist anti-CD40 antibody: a Cancer Research UK phase I study. Clin Cancer Res 21, 1321-1328

61. O'Hara MH, O'Reilly EM, Mick R et al (2019) A phase $1 \mathrm{~b}$ study of CD40 agonistic monoclonal antibody APX005M together with gemcitabine and nab-paclitaxel with or without nivolumab in untreated metastatic pancreatic ductal adenocarcinoma (PDAC) patients. Cancer Res 79 (suppl 13), abstract CT004

62. Overman MJ, LoRusso P, Strickler JH et al (2018) Safety, efficacy and pharmacodynamics (PD) of MEDI9447 (oleclumab) alone or in combination with durvalumab in advanced colorectal cancer (CRC) or pancreatic cancer (panc). J Clin Oncol 36 (suppl 15), abstract 4123

63. Siu LL, Buriss H, Le DT et al (2018) Preliminary phase 1 profile of BMS-986179, an anti-CD73 antibody, in combination with nivolumab in patients with advanced solid tumors. Cancer Res 78 (suppl 13), abstract CT180

64. Fong L, Hotson A, Powderly JD et al (2020) Adenosine $2 \mathrm{~A}$ receptor blockade as an immunotherapy for treatment-refractory renal cell cancer. Cancer Discov 10, 4053

65. Fong L, Forde PM, Powderly JD et al (2017) Safety and clinical activity of adenosine A2a receptor (A2aR) antagonist, $\mathrm{CPI}-444$, in anti-PD1/PDL1 treatment-refractory renal cell (RCC) and non-small cell lung cancer (NSCLC) patients. J Clin Oncol 35 (suppl 15), abstract 3004

66. Fong L, Chu M, George S et al (2019) Adenosine receptor blockade with Ciforadenant \pm Atezolizumab in advanced metastatic castration resistant prostate cancer (mCRPC). J Clin Oncol 38 (suppl 6), abstract 129

67. Chippori A, Williams CC, Creelan BC et al (2018) Phase I/II study of the A2AR antagonist NIR178 (PBF-509), an oral immunotherapy, in patients (pts) with advanced NSCLC. J Clin Oncol 36 (suppl 15), abstract 9089

68. Chiappori A, Creelan B, Tanvetyanon T et al (2018) Phase I/II study of the A2AR antagonist NIR178 (PBF-509) combined with the anti-PD-1 monoclonal antibody spartalizumab in patients with advanced NSCLC. Eur J Cancer 103 (suppl 1) e13-e20 O8

69. Bendell J, Bauer T, Patel $M$ et al (2019) Evidence of immunity activation in the first-in-human phase la dose escalation study of the adenosine 2a receptor antagonist, AZD4635, in patients with advanced solid tumors. Cancer Res 79 (suppl 13), abstract CT026

70. Lim EA, Bauer TM, Patel MR et al (2020) A phase I, open-label, multicenter study to assess the safety, pharmacokinetics, and preliminary antitumor activity of AZD4635 both as monotherapy and in combination in patients with advanced solid malignancies: results from prostate cancer patients. J Clin Oncol 38 (suppl 15), abstract 5518

71. Powderly JD, de Souza PL, Gutierrez R et al (2019) AB928, a novel dual adenosine receptor antagonist, combined with chemotherapy or AB122 (anti-PD-1) in patients (pts) with advanced tumors: preliminary results from ongoing phase I studies. J Clin Oncol 37 (suppl 15), abstract 2604

72. Jha GG, Gupta S, Tagawa ST et al (2019) A phase II randomized, double-blind study of sipuleucel-T followed by IDO pathway inhibitor, indoximod, or placebo in the treatment of patients with metastatic castration resistant prostate cancer (mCRPC). J Clin Oncol 35 (suppl 15), abstract 3066

73. Bahary N, Wang-Gillam A, Haraldsdottir S et al (2018) Phase 2 trial of the IDO pathway inhibitor indoximod plus gemcitabine/nab-paclitaxel for the treatment of patients with metastatic pancreas cancer. J Clin Oncol 36 (suppl 15), abstract 4015

74. Zakharia Y, Rixe O, Ward JH et al (2018) Phase 2 trial of the IDO pathway inhibitor indoximod plus checkpoint inhibition for the treatment of patients with advanced melanoma. J Clin Oncol 36 (suppl 15), abstract 9512

75. Long GV, Dummer R, Hamid O et al (2019) Epacadostat plus pembrolizumab versus placebo plus pembrolizumab in patients with unresectable or metastatic melanoma (ECHO-301/KEYNOTE- 252): a phase 3, randomised, double-blind study. Lancet Oncol 20, 1083-1097

76. Cassier PA, Italiano A, Gomez-Roca CA et al (2015) CSF1R inhibition with emactuzumab in locally advanced diffusetype tenosynovial giant cell tumours of the soft tissue: a dose-escalation and dose-expansion phase 1 study. Lancet Oncol 16, 949-956

77. Gomez-Roca CA, Italiano A, Le Tourneau C et al (2019) Phase I study of emactuzumab single agent or in combination with paclitaxel in patients with advanced/metastatic solid tumors reveals depletion of immunosuppressive M2-like macrophages. Ann Oncol 30, 1381-1392

78. Calvo A, Joensuu H, Sebastian M et al (2018) Phase Ib/lI study of lacnotuzumab (MCS110) combined with spartalizumab (PDR001) in patients (pts) with advanced tumors. 
J Clin Oncol 36 (suppl 15), abstract 3014

79. Advani R, Flinn I, Popplewell L et al (2018) CD47 blockade by Hu5F9-G4 and rituximab in non-hodgkin's lymphoma. N Engl J Med 379, 1711-1721

80. Sallmann DA, Donnellan WB, Asch AS et al (2019) The first-in-class anti-CD47 antibody Hu5F9-G4 is active and well tolerated alone or with azacitidine in $\mathrm{AML}$ and MDS patients: Initial phase $1 \mathrm{~b}$ results. J Clin Oncol 37 (suppl 15), abstract 7009

81. Sikic Bl, Lakhani N, Patnaik A et al (2019) First-in-human, first-in-class phase I trial of the anti-CD47 antibody Hu5F9G4 in patients with advanced cancers. J Clin Oncol 37, 946-953

82. Abrisqueta $P$, Sancho JM, Cordoba R et al (2019) AntiCD47 antibody, CC-90002, in combination with Rituximab in subjects with relapsed and/or refractory non-Hodgkin lymphoma (R/R NHL). Blood 134 (suppl 1), abstract 4089

83. Kim TM, Lakhani N, Gainor J et al (2019) A Phase 1 study of ALX148, a CD47 blocker, in combination with Rituximab in patients with non-Hodgkin lymphoma. Blood 134 (suppl 1), abstract 1953

84. Chow LQM, Gainor JF, Lakhani NJ et al (2020) A phase I study of ALX148, a CD47 blocker, in combination with standard anticancer antibodies and chemotherapy regimens in patients with advanced malignancy. J Clin Oncol 38 (suppl 15), abstract 3056

85. Nywening TM, Wang-Gillam A, Sanford DE et al (2016) Targeting tumour-associated macrophages with CCR2 inhibition in combination with FOLFIRINOX in patients with borderline resectable and locally advanced pancreatic cancer, a single-centre, open-label, dose-finding, nonrandomised, phase $1 \mathrm{~b}$ trial. Lancet Oncol 17, 651-662

86. Noel M, Lowery M, Ryan D et al (2017) Phase lb study of PF-04136309 (an oral CCR2 inhibitor) in combination with nab-paclitaxel/gemcitabine in first-line treatment of metastatic pancreatic adenocarcinoma. Ann Oncol 28 (suppl 5), V209-268, abstract 2138

87. Pienta KJ, Machiels JP, Schrijvers D et al (2013) Phase 2 study of carlumab (CNTO 888), a human monoclonal antibody against CC-chemokine ligand 2 (CCL2), in metastatic castration-resistant prostate cancer. Invest New Drugs 31, 760-768

88. Kim YH, Bagot M, Pinter-Brown L et al (2018) Mogamulizumab versus vorinostat in previously treated cutaneous T-cell lymphoma (MAVORIC): an international, open-label, randomised, controlled phase 3 trial. Lancet Oncol 19, 1192-1204

89. Doi T, Muro K, Ishii $\mathrm{H}$ et al (2019) A phase I study of the anti-CC chemokine receptor 4 antibody, mogamulizumab, in combination with nivolumab in patients with advanced or metastatic solid tumors. Clin Cancer Res 25, 6614-6622

90. Zamarin D, Hamid O, Nayak-Kapoor A et al (2020) Mogamulizumab in combination with durvalumab or tremelimumab in patients with advanced solid tumors: a phase I study. Clin Cancer Res 26, 4531-4541

91. Schott AF, Goldstein LJ, Cristofanilli $M$ et al (2017) Phase Ib pilot study to evaluate reparixin in combination with weekly paclitaxel in patients with HER-2-negative metastatic breast cancer. Clin Cancer Res 23, 5358-5365

92. Salgia R, Stille JR, Weaver RW et al (2017) A random- ized phase II study of LY2510924 and carboplatin/etoposide versus carboplatin/etoposide in extensive-disease small cell lung cancer. Lung Cancer 105, 7-13

93. Hainsworth JD, Reeves JA, Mace JR et al (2016) A randomized, open-label phase 2 study of the CXCR4 inhibitor LY2510924 in combination with sunitinib versus sunitinib alone in patients with metastatic renal cell carcinoma (RCC). Target Oncol 11, 643-653

94. O'Hara MH, Messersmith $\mathrm{W}$, Kindler $\mathrm{H}$ et al (2020) Safety and pharmacokinetics of CXCR4 peptide antagonist, LY2510924, in combination with Durvalumab in advanced refractory solid tumors. J Pancreat cancer 6, 21-31

95. Pernas S, Martin M, Kaufman PA et al (2018) Balixafortide plus eribulin in HER2-negative metastatic breast cancer: a phase 1, single-arm, dose-escalation trial. Lancet Oncol 19, 812-824

96. Kaufman PA, Simon SP, Martin M et al (2019) Balixafortide (a CXCR4 antagonist) + eribulin in HER2-negative metastatic breast cancer (MBC): Survival outcomes of the phase I trial. J Clin Oncol 37 (suppl 15), abstract 2606

97. Benson DM Jr, Hofmeister CC, Padmanabhan $\mathrm{S}$ et al (2012) A phase 1 trial of the anti-KIR antibody IPH2101 in patients with relapsed/refractory multiple myeloma. Blood 120, 4324-4333

98. Vey N, Bourhis JH, Boissel $\mathrm{N}$ et al (2012) A phase 1 trial of the anti-inhibitory KIR mAb IPH2101 for AML in complete remission. Blood 120, 4317-4323

99. Vey N, Goncalves A, Karlin L et al (2015) A phase 1 dose-escalation study of IPH2102 (lirilumab, BMS-986015, LIRI), a fully human anti KIR monoclonal antibody $(\mathrm{mAb})$ in patients (pts) with various hematologic (HEM) or solid malignancies (SOL). J Clin Oncol 33 (suppl 15), abstract 3065

100. Leidner R, Kang $H$, Haddad R et al (2016) Preliminary efficacy from a phase $1 / 2$ study of the natural killer cell-targeted antibody, lirilumab in combination with nivolumab in squamous cell carcinoma of the head and neck. J Immunother Cancer 4, 91

101. Bagot M, Porcu P, Marie-Cardine A et al (2019) IPH4102, a first-in-class anti-KIR3DL2 monoclonal antibody, in patients with relapsed or refractory cutaneous T-cell lymphoma: an international, first-in-human, open-label, phase 1 trial. Lancet Oncol 20, 160-170

102. Cohen RB, Bauman JR, Salas S et al (2020) Combination of monalizumab and cetuximab in recurrent or metastatic head and neck cancer patients previously treated with platinum-based chemotherapy and PD-(L) 1 inhibitors. J Clin Oncol 38 (suppl 15), abstract 6516

103. Harrington KJ, Brody J, Ingham M et al (2018) Preliminary results of the first-in-human (FIH) study of MK-1454, an agonist of stimulator of interferon genes (STING), as monotherapy or in combination with pembrolizumab (pembro) in patients with advanced solid tumors or lymphomas, Ann Oncol 29 (suppl 8), 712 abstract 5475

104. Meric-Bernstam F, Sandhu SK, Hamid O et al (2019) Phase ib study of miw815 (ADU-S100) in combination with spartalizumab (PDR001) in patients (pts) with advanced/metastatic solid tumors or lymphomas. J Clin Oncol 37 (suppl 15), abstract 2507 